\title{
Low magnetic-Prandtl number flow configurations for cold astrophysical disk models: speculation and analysis
}

\author{
O. M. Umurhan ${ }^{1,2}$ \\ 1 Astronomy Unit, School of Mathematical Sciences, Queen Mary University of London, London E1 4NS, UK \\ e-mail: umurhan@maths. qmul.ac.uk \\ 2 Astronomy Department, City College of San Francisco, San Francisco, CA 94112, USA
}

Received 24 August 2009 / Accepted 28 January 2010

\section{ABSTRACT}

\begin{abstract}
Context. Simulations of astrophysical disks in the shearing box that are subject to the magnetorotational instability (MRI) show that activity appears to be reduced as the magnetic Prandtl number $P_{\mathrm{m}}$ is lowered. It is therefore important to understand the reasons for this trend, especially if this trend is shown to continue when higher resolution calculations are performed in the near future. Calculations for laboratory experiments show that saturation is achieved through modification of the background shear for $P_{\mathrm{m}} \ll 1$.

Aims. Guided by the results of calculations appropriate for laboratory experiments when $P_{\mathrm{m}}$ is very low, the stability of inviscid disturbances in a shearing box model immersed in a constant vertical background magnetic field is considered under a variety of shear profiles and boundary conditions in order to evaluate the hypothesis that modifications of the shear bring about saturation of the instability. Shear profiles $q$ are given by the local background Keplerian mean, $q_{0}$, plus time-independent departures, $Q(x)$, with zero average on a given scale.

Methods. The axisymmetric linear stability of inviscid magnetohydrodynamic normal modes in the shearing box is analyzed.

Results. (i) The stability/instability of modes subject to modified shear profiles may be interpreted by a generalized Velikhov criterion given by an effective shear and radial wavenumber that are defined by the radial structure of the mode and the form of $Q$. (ii) Where channel modes occur, comparisons against marginally unstable disturbance in the classical case, $Q=0$, shows that all modifications of the shear examined here enhance mode instability. (iii) For models with boundary conditions mimicing laboratory experiments, modified shear profiles exist that stabilize a marginally unstable MRI for $Q=0$. (iv) Localized normal modes on domains of infinite radial extent characterized by either single defects or symmetric top-hat profiles for $Q$ are also investigated. If the regions of modified shear are less (greater) than the local Keplerian background, then there are (are no) normal modes leading to the MRI.

Conclusions. The emergence and stability of the MRI is sensitive to the boundary conditions adopted. Channel modes do not appear to be stabilized through modifications of the background shear whose average remains Keplerian. However, systems that have nonpenetrative boundaries can saturate the MRI through modification of the background shear. Conceptually equating the qualitative results from laboratory experiments to the conditions in a disk may therefore be misleading.
\end{abstract}

Key words. accretion, accretion disks - magnetohydrodynamics (MHD) - instabilities - protoplanetary disks

\section{Introduction}

After almost 40 years of investigation the source of the anomalous transport in accretion disks still remains an open question. As it is mostly assumed that the transport is a reflection of some underlying turbulent state, identification of its source mechanism and process has been the focus of much research activity. A leading candidate mechanism is the magnetorotational instability (Balbus \& Hawley 1991).

The magnetic Prandtl number $\left(P_{\mathrm{m}}\right)$, i.e. the ratio of a fluid's viscosity to its magnetic diffusivity, appears to play an important role in the ability of the MRI to drive a fully-developed turbulent state. Recently resolved simulations of the MRI in a shearing box environment (e.g. Lesur \& Longaretti 2007; Fromang et al. 2008) show the appearance of a turbulent state for moderately high Reynolds numbers $(\mathrm{Re})$ when $P_{\mathrm{m}}$ is an order 1 quantity or higher. However, the amount of transport delivered by the MRI appears to depend on $P_{\mathrm{m}}$ : as this quantity decreases the vigor of the turbulent state decreases and if $P_{\mathrm{m}}$ drops below some critical value, then the turbulence appears to vanish altogether.

Estimates of the properties of cold astrophysical disks, like protoplanetary disks, show that their characteristic $P_{\mathrm{m}}$ 's are on the order of $10^{-6}$ at best and that the MRI may be operating only in the disk's corona as its ionization fraction is sufficient to merit an MHD description there rather than near the disk midplane (see Balbus \& Henri 2008, and references therein). Thus, the fate of the MRI in disk environments, in which the magnetic Prandtl number is characteristically small, remains to be settled.

It is not clear why turbulent transport appears to vanish in numerical experiments when $P_{\mathrm{m}}$ weakens. One possibility may be that under those conditions the MRI cannot grow sufficiently to excite a secondary transition that would, in turn, open the way to a turbulent cascade. Theoretical considerations of the MRI for restrictive configurations like laboratory experiments with cylindrical geometry predict that a low $P_{\mathrm{m}}$ sheared fluid undergoing the MRI settles onto a pattern state whose momentum transport scales as $\sim\left(P_{\mathrm{m}}\right)^{\alpha}$ where $\alpha \geq 1-$ in other words, saturation of the instability is sensitive to the microscopic viscosity of the fluid. This counterintuitive result is rationalized by noting that a weak fluid viscosity means that it takes very little effort to readjust the underlying flow profile and establish a new shear in which the instability cannot operate. The stronger the viscosity, then, it becomes more difficult for the fluid to shut off the shear and the instability may operate unabatedly during the fluid's nonlinear 
development and eventual cascade to turbulence via secondary instabilities (e.g. parasitic instabilities, Xu \& Goodman 1994).

In the idealized quasi-linear study of Knobloch \& Julien (2006) it was shown that in the limit of very large hydrodynamic and magnetic Reynolds numbers (i.e. $\operatorname{Re} \gg 1$ and $\operatorname{Re}_{\mathrm{m}} \gg 1$ respectively) the azimuthal velocity profile tips over to shut off the instability by establishing a new velocity profile which reduces the shear throughout the domain. Their analysis shows that in the limits where $\operatorname{Re} \rightarrow \infty$ and $\operatorname{Re}_{\mathrm{m}} \rightarrow \infty$ the resulting net velocity profile tends toward zero shear. Examinations of the thin-gap Taylor-Couette system in the low $P_{\mathrm{m}}$ limit (Umurhan et al. 2007a,b) show that once the system reaches saturation, the resulting velocity field within large mid-portions of the experimental domain is characterized by weakened shear. However these regions, where the tendency toward instability is reduced, are sandwiched by regions of strengthened shear, where the tendency toward instability is enhanced. Nonetheless, the aggregate configuration results in a profile that is stable against the MRI or any secondary instability and the system settles onto nonturbulent pattern state. Equally relevant is that in this pattern state the amplitudes of all fluid quantities, except the modified shear, scale as some power of $P_{\mathrm{m}}$. By comparison the modification of the shear is an order 1 quantity. Thus, in the limit where $P_{\mathrm{m}}$ becomes very small only the modification to the background shear appears as a noticeable response in the idealized studies of the laboratory setup.

Because resolved shearing box numerical experiments of high $\mathrm{Re}$ and low $P_{\mathrm{m}}$ flows appropriate for cold astrophysical disks is currently out of reach, speculation at this stage is justifiable. In particular, what may be the effective azimuthal velocity profile of high $\mathrm{Re}$ and low $P_{\mathrm{m}}$ disks (or their sections)? As Ebrahimi et al. (2009) recently note, quasi-linear saturation of the shear profile might be an unreasonable expectation given that the strong gravity tends to restore the shear profile within disks. However, the theoretical results of the thingap Taylor-Couette system and the quasi-linear studies, together with the objection of Ebrahimi et al., point to a possible hybrid scenario: what if the velocity profile of a low $P_{\mathrm{m}}$ disk is on average Keplerian but locally exhibits alternating zones of very weak (or no) shear and very strong shear? This hypothetical configuration of the azimuthal velocity profile, an example of which is depicted in Fig. 1, might very well be driven into place by the MRI under very low $P_{\mathrm{m}}$ situations. The recent studies of Julien $\&$ Knobloch (2006) and Jamroz et al. (2008) argue for the relevance of this scenario.

This study is an examination of the axisymmetric linear stability of incompressible ideal magnetohydrodynamic disturbances in a shearing box threaded by a constant vertical magnetic field and characterized by a variety of mean velocity profiles, including an extreme instance of the one appearing in Fig. $1^{1}$. Such configurations might be representative of real astrophysical disks characterized by very small values of $P_{\mathrm{m}}$.

Guided by the low $P_{\mathrm{m}}$ results of Knobloch \& Julien (2005) and Umurhan et al. (2007a,b), the analysis undertaken in this study is inviscid. The only low $P_{\mathrm{m}}$ effect that is included here is the possibility that the fundamental shear profile is significantly modified. This altered shear profile may be easily included into the equations of motion as any radially dependent barotropic steady shear profile is a permitted equilibrium flow solution of the small shearing-box equations.

\footnotetext{
${ }^{1}$ Extreme in the sense that regions of sharp shear are represented by delta-functions in the analysis herein. See also discussion in Sect. 6.
}

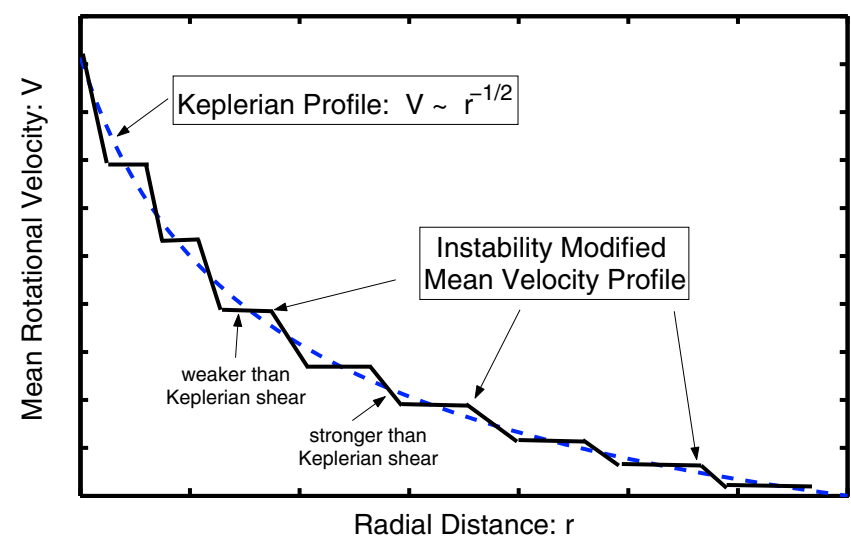

Fig. 1. Qualitative depiction of a hypothesized mean rotational velocity profile for a low magnetic Prandtl number disk suggested by Jamroz et al. (2008). The dashed line shows the usual Keplerian velocity profile describing a rotationally supported disk. The mean velocity profile resulting from the saturation of the MRI in the low $P_{\mathrm{m}}$ limit is speculated to consist of alternating regions of low and high shear (black lines). The mean shear over the extent of the disk follows the general Keplerian profile.

The range of questions that figure prominently are: (i) what happens to the unstable mode leading to the MRI when there are departures of the steady shear from the background Keplerian state? (iii) How does the nature or existence of unstable modes depend upon the radial boundary conditions of the shearing box (e.g. whether it is open or periodic in the radial direction)? (ii) Can one infer a possible reason or explanation for why turbulence seems to vanish in numerical experiments of the shearing box when $P_{\mathrm{m}}$ is small?

This work is organized as follows: in Sect. 2 the equations of motion are laid out and the framework within which the stability analysis is discussed. Section 3 develops the profiles for the steady state for given modifications of the background shear while in Section 4 the normal mode stability analysis is developed and the governing ODE is derived including a statement of the generalized Velikhov criterion (Velikhov 1959). Section 5 presents the results of the stability analysis for a variety of boundary conditions and shear profiles and their forms. The implications of the results are discussed in the final section.

\section{Equations of Motion}

The small shearing box equations (SSB, Lesur \& Longaretti 2007; Regev \& Umurhan 2008) are adopted which are the incompressible incarnation of the usual shearing box equations (Goldreich \& Lynden-Bell 1965). Their usage is justified as compressibility is an inessential feature for the MRI. The box is moving in a rotating frame moving with the local Keplerian velocity $\bar{V}_{K}{ }^{2}$. The length scales of the box are measured by a size $\bar{L}$ which is much smaller than the radial scale of the disk $\bar{R}_{0}$. On these scales, the background Keplerian shear appears as a linear Couette flow profile. Time units are scaled by the local rotation time of the disk $\bar{\Omega}\left(\bar{R}_{0}\right)$, as measured at the fiducial radius $\bar{R}_{0}$, implying that the velocities of interest are scaled by $\bar{U}_{0}=\bar{L} \bar{\Omega}\left(\bar{R}_{0}\right)$. Given this, the disparity of the ratio of the local disk soundspeed to the Keplerian speed (since the gas is relatively cold), and the assumption that the scale $\bar{L}$ is much less than the vertical scale height of the disk, the flow dynamics have the character of incompressible rotating magnetic Couette flow.

\footnotetext{
${ }^{2}$ Overbars over quantities refer to their dimensional values.
} 
Magnetic fields are scaled by a reference field scale $\bar{B}_{0}$ and the density is $\bar{\rho}$. These quantities form a velocity defined to be the Alfven speed via $\bar{U}_{A}^{2} \equiv \bar{B}_{0}^{2} / 4 \pi \bar{\rho}$. Furthermore the Cowling number is defined

$C \equiv \frac{\bar{U}_{A}^{2}}{\bar{U}_{0}^{2}}$

which may be considered as related to the inverse-square of the " $\beta$-parameter" of plasma physics. This is not to be confused with the parameter $\beta$ used later on in this work to designate radial wavenumbers. The non-dimensionalized SSB equations of motion are

$\left(\partial_{t}+\boldsymbol{u} \cdot \nabla\right) \boldsymbol{u}+2 \Omega_{0} \hat{z} \times \boldsymbol{u}=-\frac{1}{\rho} \nabla p+2 \Omega_{0}^{2} q_{0} x \hat{\boldsymbol{x}}+C \boldsymbol{J} \times \boldsymbol{B}$,

$\nabla \cdot \boldsymbol{u}=0$

$\partial_{t} \boldsymbol{B}=\nabla \times \boldsymbol{u} \times \boldsymbol{B}$,

$\boldsymbol{J}=\nabla \times \boldsymbol{B}$,

in which $\boldsymbol{u}$ is the velocity vector, $p$ is the pressure, $\rho$ is the constant density (which is 1 in these units). $\Omega_{0}$ is the local rotation rate but in the units used here it is also 1 . The parameter $q_{0}$ defines the amplitude of the local Keplerian shear and is formally given by

$q_{0} \equiv-\left(\frac{\bar{R}}{\bar{\Omega}} \cdot \frac{\partial \bar{\Omega}}{\partial \bar{R}}\right)_{\bar{R}_{0}}$,

which is equal to $3 / 2$ for rotationally supported Keplerian disks. This is the value assumed for $q_{0}$ throughout the remainder of this work. The magnetic field is given by the vector $\boldsymbol{B}$ and the current $\boldsymbol{J}$ and is governed by Ampere's $\mathrm{Law}^{3}$.

\section{Steady state}

It is assumed that the fluid is of constant density $\rho_{0}$ and the fluid is threaded uniformly throughout by a constant vertical magnetic field, i.e. $\boldsymbol{B}=B_{z} \hat{\boldsymbol{z}}$. The current source of this background field is taken to be external to the domain. As stated in the Introduction the steady velocity field that represent departures to the Keplerian shear are taken as given and represented by $U(x)$. The total mean velocity is written as $V(x)$ which is the sum of the Keplerian portion $-q_{0} \Omega x$ and the departures $U$. Thus the steady state configuration requires satisfying the radial momentum balance which becomes

$-2 \Omega_{0}\left(-q_{0} \Omega_{0} x+U\right)=-\partial_{x} \varpi_{0}+2 \Omega_{0}^{2} q_{0} x$,

in which the total mechanical and magnetic pressure is given by

$\varpi_{0}=p_{0} / \rho_{0}+\frac{1}{2} C B_{z}^{2}$,

where $p_{0}$ is the steady state mechanical pressure field. For the model analyzed here where $B_{z}$ is constant, the steady pressure field $p_{0}$ is related to the azimuthal velocity departures by

$\partial_{x} p_{0}=2 \Omega_{0} U \bar{\rho}$.

The above expression represents a radially geostrophic state. Note that the solutions to $p_{0}$ are smooth and well behaved so long as $\tilde{V}$ is continuous across the domain. In the axisymmetric

\footnotetext{
${ }^{3}$ Note that by its construction (3) contains the statement that the time evolution of the divergence of the magnetic field is zero. If initially $\nabla \cdot \boldsymbol{B}=0$ everywhere, then it remains identically zero subsequently.
}

linear stability analysis that follows only the steady state total vorticity field, denoted by $q$, plays a role and it is defined as

$q=q_{0}+Q$

where $Q(x) \equiv-\partial_{x} U / \Omega_{0}$. A variety of functional forms for $Q$ will be examined. The only restriction on $Q$ will be that its domain integral is bounded, i.e.,

$\left|\int_{\mathcal{D}} Q \mathrm{~d} x\right|<\infty$

where $\mathcal{D}$ symbolizes the domain under consideration. In many cases this integral will be zero.

\section{Linear theory}

Axisymmetric infinitesimal disturbances about the steady state shaped by $q(x)$ are introduced into the governing equations of motion $(1-4)$ revealing,

$\partial_{t} u^{\prime}-2 \Omega_{0} v^{\prime}=-\partial_{x}\left(p^{\prime} / \rho_{0}+C B_{z} b_{z}^{\prime}\right)+C B_{z} \partial_{z} b_{x}^{\prime}$,

$\partial_{t} v^{\prime}+(2-q) \Omega_{0} u^{\prime}=C B_{z} \partial_{z} b_{y}^{\prime}$,

$\partial_{t} w^{\prime}=-\partial_{z}\left(p^{\prime} / \rho_{0}+C B_{z} b_{z}^{\prime}\right)+C B_{z} \partial_{z} b_{z}$,

$\partial_{x} u^{\prime}+\partial_{z} w^{\prime}=0$

$\partial_{t} b_{x}^{\prime}=B_{z} \partial_{z} u$,

$\partial_{t} b_{y}^{\prime}=-\Omega_{0} q b_{x}+B_{z} \partial_{z} v^{\prime}$

$\partial_{t} b_{z}^{\prime}=B_{z} \partial_{z} w^{\prime}$

in which $u^{\prime}, v^{\prime} w^{\prime}$ and $p^{\prime}$ denote perturbations in the radial, azimuthal vertical velocities and pressures, respectively, while $b_{x, y, z}^{\prime}$ represent perturbations in the magnetic field components. It should be recalled that $q(x)$ is unspecified at this stage. However the existence of a steady state is assumed for a given reasonable $q(x)$ profile. Normal mode perturbations are assumed which are periodic in the vertical direction. A perturbation variable $f^{\prime}(x, z, t)$ is thus expressed via the ansatz $f^{\prime}(x) \exp (\sigma t+$ $\mathrm{i} k z$ ) where $\sigma$ is the temporal response while $k$ is the vertical wavenumber. The incompressibility condition (11) means that a streamfunction $\psi^{\prime}$ may be defined such that,

$u^{\prime}=\partial_{z} \psi^{\prime}, \quad w^{\prime} \equiv-\partial_{x} \psi^{\prime}$.

Similarly the expression (12) and (14) allow for the definition of a flux function $\Phi$ in which the radial and vertical components of the perturbed magnetic field are expressed via $b_{x}=\partial_{z} \Phi^{\prime}$ and $b_{z} \equiv-\partial_{x} \Phi^{\prime}$.

Utilizing these definitions, the linearized perturbation equations are reduced to a single one for the streamfunction (wherein and henceforth the primes are dropped),

$\partial_{x}^{2} \psi-\kappa^{2} \psi=0$

in which

$\kappa^{2} \equiv k^{2}\left(1+\frac{\omega^{2} \sigma^{2}-\Omega_{0}^{2} 2 q \omega_{a z}^{2}}{\left(\sigma^{2}+\omega_{a z}^{2}\right)^{2}}\right) ; \quad \omega_{a z}^{2} \equiv C B_{z}^{2} k^{2}$,

where $\omega_{a z}$ is the Alfven frequency and the spatially dependent epicyclic frequency is defined by $\omega(x)^{2} \equiv 2(2-q) \Omega_{0}^{2}$. For later usage $\kappa$ is rewritten in the alternate form,

$\kappa^{2}=k^{2}\left(1+\frac{4 \Omega_{0}^{2} \sigma^{2}}{\left(\sigma^{2}+\omega_{a z}^{2}\right)^{2}}-\frac{2 \Omega_{0}^{2} q}{\sigma^{2}+\omega_{a z}^{2}}\right)$. 
The solutions to this system is governed by several parameters including the vertical wavenumber $k$, the Cowling number, $C$, the radial size of the domain (appearing below) $L$ and the form and amplitude of the deviation shear profile $Q$. Taken as a representation of a small disk section it is assumed that there exists a minimum vertical wavenumber $k_{0}$ for disturbances since it is not reasonable to consider fundamental disturbances whose vertical extent is much larger than the disk height itself (Curry et al. 1994, and see discussion).

Before proceeding to these results it is important to keep in mind a number of matters. First, Knobloch (1992) showed that for vertical field configurations like that considered here, together with boundary conditions in which individual disturbances or their combinations are zero on the boundaries, the character of the normal mode response of the MRI is that $\sigma$ is either real (growing/decaying exponential modes) or imaginary (oscillating modes), but never complex ${ }^{4}$. A version of the argument leading to this conclusion is given in Appendix A. Thus when the character of the fluid response is analyzed, $\sigma^{2}$ will be taken as $\in$ Reals. The relevance of this observation is that as a function of the system's parameters, a mode becomes unstable by passing through $\sigma^{2}=0$, sometimes known as exchange of stabilities (Chandrasekhar 1961). As such, in some instances the stability analysis of this system will focus on identifying which mode becomes marginal (i.e. $\sigma^{2}=0$ ) and under which parameter conditions.

Secondly, the very same integral argument developed in Appendix A also leads to a general statement about the relationship between the temporal response and the structure of the modes in the domain,

$1+\frac{\bar{\beta}^{2}}{k^{2}}+\frac{4 \Omega_{0}^{2} \sigma^{2}}{\left(\sigma^{2}+\omega_{a z}^{2}\right)^{2}}-\frac{2 \Omega_{0}^{2} \bar{q}}{\sigma^{2}+\omega_{a z}^{2}}=0$.

$\bar{\beta}$ is like an average radial wavenumber of the disturbance,

$\bar{\beta}^{2} \equiv \frac{\int_{\mathcal{D}}\left|\partial_{x} \psi\right|^{2} \mathrm{~d} x}{\int_{\mathcal{D}}|\psi|^{2} \mathrm{~d} x}$,

and, evidentally, $\bar{\beta}^{2}$ is always greater than zero. $\bar{q}$ represents the mode weighted average shear over the domain

$\bar{q} \equiv q_{0}+\frac{\int_{\mathcal{D}} Q|\psi|^{2} \mathrm{~d} x}{\int_{\mathcal{D}}|\psi|^{2} \mathrm{~d} x}$.

One immediate consequence of this is that if $\bar{q}=0$ (i.e. the mode weighted average shear over the domain is zero), then there is no possibility of instability. This follows from evaluating the criterion for marginality in (18), setting $\sigma^{2}$ to zero and revealing

$1+\frac{\bar{\beta}^{2}}{k^{2}}-\frac{2 \Omega_{0}^{2} \bar{q}}{\omega_{a z}^{2}}=0$.

The above expression cannot be satisfied if $\bar{q}$ is zero. In general this relationship is not useful for direct calculations as the quantities therein depend implicitly upon the eigenvalue $\sigma$. However, it will be called upon to aid in interpreting the results that are obtained from specific direct calculations in Sect. 6.

\footnotetext{
${ }^{4}$ It was also shown in that study that complex values of $\sigma$ can only occur if the field configuration is helical. In this case the instability is of a traveling wave.
}

\section{Results}

\subsection{Classical MRI modes and channel solutions: a review}

The classical system is recovered for $Q=0$. This means that $\kappa$ is independent of $x$ and

$\kappa^{2} \rightarrow \kappa_{0}^{2} \equiv k^{2}\left(1+\frac{4 \Omega_{0}^{2} \sigma^{2}}{\left(\sigma^{2}+\omega_{a z}^{2}\right)^{2}}-\frac{2 \Omega_{0}^{2} q_{0}}{\sigma^{2}+\omega_{a z}^{2}}\right)$.

Solutions of (15) are sought on a periodic domain $L$ and are given in general by

$\psi=A \cosh \left(\kappa_{0} x+\phi\right)$

where $A$ and $\phi$ are constants set by the boundary conditions of the system. The periodicity condition that $\psi(x+L / 2)=$ $\psi(x-L / 2)$ then means that

$1-\cosh \left[L \kappa_{0}\right]=0$.

which has the solution $\kappa_{0} L=2 n i \pi$ where $n$ is any integer including zero. This condition recovers the incompressible limit of the classical dispersion relationship of the ideal case (e.g. Acheson \& Hide 1973; Balbus \& Hawley 1991) where specifically,

$$
\left(\beta_{n}^{2}+k^{2}\right)\left(\sigma^{2}+\omega_{a z}^{2}\right)^{2}+\omega_{0}^{2} k^{2} \sigma^{2}-2 \Omega_{0}^{2} q_{0} k^{2} \omega_{a z}^{2}=0 .
$$

in which $\omega_{0}^{2} \equiv 2\left(2-q_{0}\right) \Omega_{0}^{2}$ is the epicyclic frequency and where the radial wavenumber $\beta_{n}$ is

$\beta_{n}^{2} \equiv \frac{4 n^{2} \pi^{2}}{L^{2}}$

The solutions for the temporal response, written in terms of respected pairs, is given by

$$
\begin{aligned}
\sigma^{2}= & \sigma_{0 n}^{2}\left(\beta_{n}^{2}\right) \equiv-\left(\omega_{a z}^{2}+\frac{k^{2}}{\beta_{n}^{2}+k^{2}} \frac{\omega_{0}^{2}}{2}\right) \\
& \pm\left[\left(\omega_{a z}^{2}+\frac{k^{2}}{\beta_{n}^{2}+k^{2}} \frac{\omega_{0}^{2}}{2}\right)^{2}-\omega_{a z}^{2}\left(\omega_{a z}^{2}-\frac{2 \Omega_{0}^{2} q_{0} k^{2}}{\beta_{n}^{2}+k^{2}}\right)\right]^{1 / 2}
\end{aligned}
$$

The solutions associated with the "-" branch are termed the hydrodynamic inertial (HI) modes while those associated with the "+" are the hydromagnetic inertial (HMI) modes (viz. Acheson $\&$ Hide 1973). For a given mode $n$, the magnetorotational instability occurs for the latter of these provided that $\omega_{0}^{2}>0$ and

$\omega_{a z}^{2}-\frac{2 \Omega_{0}^{2} q_{0} k^{2}}{\beta_{n}^{2}+k^{2}}<0$.

The background field must be sufficiently weak for the HMI-modes to be unstable.

For given values of $\omega_{a z}^{2}$ the absolute minimum criterion required for instability to set in (i.e. for the mere possibility of $\sigma^{2}>0$ ) is when $n=0$, i.e. $\kappa_{0}=0$, and the condition in (27) predicts this to happen if,

$2 \Omega_{0}^{2} q_{0}-\omega_{a z}^{2}>0$

(Velikhov 1959; Acheson \& Hide 1973). This particular special mode, in which its radial structure is uniform, is generally referred to in the literature as the channel mode which describes 
purely 2D flow with no vertical velocity. The temporal response in this case is given by $\sigma_{0 n}^{2}=\sigma_{00}^{2}$ in which,

$$
\begin{aligned}
\sigma_{00}^{2}= & -\left(\omega_{a z}^{2}+\frac{\omega_{0}^{2}}{2}\right) \\
& \pm\left[\left(\omega_{a z}^{2}+\frac{\omega_{0}^{2}}{2}\right)^{2}-\omega_{a z}^{2}\left(\omega_{a z}^{2}-2 \Omega_{0}^{2} q_{0}\right)\right]^{1 / 2} .
\end{aligned}
$$

When unstable (for the HMI-mode branch) this radially uniform mode plays the central role in the development of MRI induced turbulence in numerical experiments. If $2-q_{0}>0$, then the expression for $\sigma_{00}^{2}$ is

$$
\begin{aligned}
\sigma_{00}^{2}+ & \omega_{a z}^{2}=-\Omega_{0}^{2}\left(2-q_{0}\right) \\
& \quad \pm \Omega_{0}^{2}\left(2-q_{0}\right)\left[1+\frac{4 \omega_{a z}^{2}}{\Omega_{0}^{2}\left(2-q_{0}\right)}+\frac{8 \Omega_{0}^{2} q_{0} \omega_{a z}^{2}}{\Omega_{0}^{4}\left(2-q_{0}\right)^{2}}\right]^{1 / 2} .
\end{aligned}
$$

The combination expression $\sigma_{00}^{2}+\omega_{a z}^{2}$ (which shall appear on a number of occasions in the following discussion) is always positive for HMI modes and is always negative for the HM modes since the expression inside the square-root operation is always positive.

It should be noted that for $Q=0$ there are no localized normal modes possible for a domain which is infinite in extent. Put in another way, there is no solution of (15) for modes on an infinite domain with both (a) all quantities going to zero as $x \rightarrow \pm \infty$ and (b) $\kappa$ is constant as given in (22).

\subsection{Weak shear variations on a finite domain: $0<Q \ll 1$}

In this section weak shear variations are considered whose average are zero on the domain $L$. Before proceeding a caveat ought to be stated. Despite the spatially periodic nature of the shear $Q$, the analysis necessary to determine the temporal response uses a multiple time-scale analysis. When periodic boundary conditions are imposed, as they are in Sect. 5.2.3, the multiple timescale analysis is akin to a Floquet analysis. In this instance a long-time scale behavior (in the form of correspondingly weak corrections to the temporal response $\sigma$ ) must be invoked in order to ensure that the resulting perturbation solutions remain periodic on the length scale $L$. For boundary conditions other than periodic ones (Sects. 5.2.1 and 5.2.2), the multiple-time scale analysis employed is a generic procedure involving perturbation series expansions and imposition of solvability conditions at successive perturbation orders (Bender \& Orszag 1999). The role of the solvability conditions is to make sure that higher order perturbation solutions satisfy the boundary conditions of the system.

The shear with zero average on the domain of length $L$ may be decomposed into the Fourier series expansion

$$
Q=\epsilon Q_{1} ; \quad Q_{1}=\sum_{n=1}^{\infty} q_{n} \sin \left(\frac{2 n \pi}{L} x-\frac{\pi}{2}\right),
$$

where the parameter $\epsilon \ll 1$ measures the overall severity of the shear. $q_{n}$ measures the Fourier amplitude of the shear component $n$ and is treated hereafter as a tunable parameter. Reference to (6) indicates that this form for $Q$ ensures that the deviation steady velocity $U$ and, hence, the radial pressure gradients are zero at the boundaries $x= \pm L / 2$. The governing equation, with $Q$ as given above, is expressed as

$\partial_{x}^{2} \psi-\kappa_{0}^{2} \psi=-\epsilon \frac{2 \Omega_{0}^{2} k^{2} Q_{1}}{\sigma^{2}+\omega_{a z}^{2}} \psi$.
Solutions of this are developed in a singular perturbation series expansion in powers of $\epsilon$. Thus the ansatz,

$$
\begin{aligned}
& \psi=\psi_{0}+\epsilon \psi_{1}+\epsilon^{2} \psi_{2}+\cdots \\
& \sigma^{2}=\sigma_{0}^{2}+\epsilon \sigma_{1}^{2}+\epsilon^{2} \sigma_{2}^{2}+\cdots \\
& \kappa_{0}^{2}=\kappa_{00}^{2}+\epsilon \kappa_{01}^{2}+\epsilon^{2} \kappa_{02}^{2}+\cdots
\end{aligned}
$$

Given the definition of $\kappa_{0}^{2}$ given in (22) it follows that $\kappa_{00}^{2}=$ $\kappa_{0}^{2}\left(\sigma_{0}^{2}\right)$ and

$$
\begin{aligned}
& \kappa_{01}^{2} \equiv\left(\frac{\partial \kappa_{0}^{2}}{\partial \sigma^{2}}\right)_{\sigma_{0}^{2}} \sigma_{1}^{2} \\
& \kappa_{02}^{2} \equiv \frac{1}{2}\left(\frac{\partial^{2} \kappa_{0}^{2}}{\partial \sigma^{4}}\right)_{\sigma_{0}^{2}} \sigma_{1}^{4}+\left(\frac{\partial \kappa_{0}^{2}}{\partial \sigma^{2}}\right)_{\sigma_{0}^{2}} \sigma_{2}^{2},
\end{aligned}
$$

wherein

$$
\left(\frac{\partial \kappa_{0}^{2}}{\partial \sigma^{2}}\right)=\frac{k^{2}}{\sigma^{2}+\omega_{a z}^{2}}\left(-\frac{\omega_{0}^{2}}{\sigma^{2}+\omega_{a z}^{2}}+\frac{8 \Omega_{0}^{2} \omega_{a z}^{2}}{\left(\sigma^{2}+\omega_{a z}^{2}\right)^{2}}\right) .
$$

The possibility that $\sigma_{1}^{2}=0$ must be allowed (see below) This is why the next order term is also included in the expansion. With these expansions in mind (15) is written out for each power of $\epsilon$. To lowest order

$\partial_{x}^{2} \psi_{0}-\kappa_{00}^{2} \psi_{0}=0$

and to order $\epsilon$

$\partial_{x}^{2} \psi_{1}-\kappa_{00}^{2} \psi_{1}=\left(\kappa_{01}^{2}-\frac{2 \Omega_{0}^{2} k^{2} Q_{1}}{\sigma_{0}^{2}+\omega_{a z}^{2}}\right) \psi_{0}$.

The order $\epsilon^{2}$ form is relegated until later. Solutions to $(36,37)$ are developed in the following subsections for various boundary conditions and restrictions at $x= \pm L / 2$.

\subsubsection{Channel conditions}

Channel conditions require that the perturbed radial velocity is zero at the boundaries $x= \pm L / 2$. This, in turn, amounts to requiring that $i k \psi=0$ at these positions, or in terms of the perturbation variables

$\psi_{0}=\psi_{1}=0, \quad$ at $x= \pm L / 2$.

The lowest order solution is given by

$\psi_{0}=\sum_{m=1}^{\infty} \psi_{0 m}=\sum_{m=1}^{\infty} A_{m} \sin \gamma_{m}(x+L / 2) ; \quad \gamma_{m} \equiv \frac{m \pi}{L}$

and where $\kappa_{00}^{2}=-\gamma_{m}^{2}$. The general solution for $\psi_{0}$ is written out as a sum of the linearly independent solutions $\psi_{0 m}$. The index $m$ ought not be confused with the index $n$ which refers to the Fourier component of the shear profile $Q$ appearing in (31). Stability, on the other hand, is evaluated by examining each individual eigenmode $m$. Thus all eigenvalues $\sigma$ (and their perturbative corrections) will be identified according to which eigenmode under examination, e.g. $\sigma_{0 m}, \sigma_{1 m}$ etc. Note also that here the index $m$ does not include $m=0$ as this would correspond to the trivial state $\psi=0$.

Given the functional form of $\kappa_{0}^{2}$ found in (22), these relationships imply that $\sigma_{0}^{2}$ is equal to the functional form $\sigma_{0 n}^{2}$ found in (26) except with $\beta_{n}$ replaced by $\gamma_{m}$, i.e. $\sigma_{0}^{2}=\sigma_{0 n}^{2}\left(\gamma_{n}^{2}\right)$. The stability of these modes is now a function of the mode number $m$ 
and, henceforth, the summation sign in (38) will be dropped and it shall be henceforth understood that when reference is made to $\psi_{0}$, a particular eigenmode mode $m$ will be analyzed. At the next order it can be seen that in order to have solutions that satisfy the boundary condition that $i k \psi_{1}=0$ at the two endpoints, the terms on the RHS of (37) must satisfy a particular solvability condition which will relate the correction $\sigma_{1}$ to the variation $Q_{1}$. This is seen by multiplying (37) by $\psi_{0}$ given in (38) and integrating the result across the domain from $-L / 2$ to $L / 2^{5}$. In a general sense, then, one finds,

$\kappa_{01}^{2}=\left(\frac{\partial \kappa_{0}^{2}}{\partial \sigma^{2}}\right)_{\sigma_{0 m}^{2}} \sigma_{1 m}^{2}=\frac{2 \Omega_{0}^{2} k^{2}}{\sigma_{0 m}^{2}+\omega_{a z}^{2}} \frac{\left\langle Q_{1} \psi_{0 m}^{2}\right\rangle}{\left\langle\psi_{0 m}^{2}\right\rangle}$,

in which the bracket notation is

$\langle\diamond\rangle \equiv \int_{-L / 2}^{L / 2} \diamond \mathrm{d} x$.

It follows that

$\sigma_{1 m}^{2}=-q_{m}(-1)^{m} \Upsilon(m)$,

in which

$\Upsilon(m) \equiv \frac{\Omega_{0}^{2}\left(\sigma_{0 m}^{2}+\omega_{a z}^{2}\right)^{2}}{-\omega_{0}^{2} \sigma_{0 m}^{2}+2 \Omega_{0}^{2}\left(2+q_{0}\right) \omega_{a z}^{2}}$.

The denominator term of $\Upsilon(m)$ never crosses zero for physically relevant values of the parameters so that $\Upsilon>0$.

For illustration consider the $m=1$ mode (which is also the most unstable one) near marginality in the limit where $k L$ is very large. In order for $\sigma_{01}^{2}$ to be nearly zero it must be that $\omega_{a z}^{2} \approx 2 \Omega_{0}^{2} q_{0}$, cf. (28). It follows that the correction for this mode, $\sigma_{11}$, is given approximately by the solution to

$\sigma_{11}^{2} \approx q_{1} \frac{\omega_{a z}^{2}}{2\left(2+q_{0}\right)}=q_{1} \frac{\Omega_{0}^{2} q_{0}}{\left(2+q_{0}\right)}$,

where the last equality is established because the marginal mode, $\sigma_{01}^{2} \approx 0$ is under examination. The introduction of this profile pattern stabilizes this otherwise marginal mode if $q_{1}<0$ and destabilizes it when $q_{1}>0$. The corresponding shear profile for this example is depicted in Fig. 2. It can be seen that the modified shear is stabilizing when the shear is positive near the boundaries and negative in the interior.

\subsubsection{Pressure conditions}

An analysis of (10) and (14) shows that the total magnetic plus mechanical pressure perturbations are proportional to the radial gradient of $\psi$ i.e.,

$p / \rho_{0}+B_{z} C b_{z}=\frac{\sigma^{2}+\omega_{a z}^{2}}{\sigma} \partial_{x} \psi$

Fixing the total pressure at the boundary in a Lagrangian sense (Curry et al. 1994) is equivalent to setting to zero the above expression at $x= \pm L / 2$ which, in the present case, means setting $\partial_{x} \psi=0$ at those locations ${ }^{6}$. The solution $\psi_{0 m}$ is then given by

$\psi_{0 m}=A_{m} \cos \gamma_{m}(x+L / 2) ; \quad \gamma_{m} \equiv \frac{m \pi}{L}$,

5 This procedure of applying a solvability condition actually requires multiplying across by the adjoint solution of $\psi_{0}$. However since the eigenfunctions comprising $\psi_{0}$ and the linear operator of (36) are real and Hermitian, the adjoint is $\psi_{0}$.

${ }^{6}$ Note that this is valid since by construction the radial gradient of the steady state pressure field is zero at the end points.

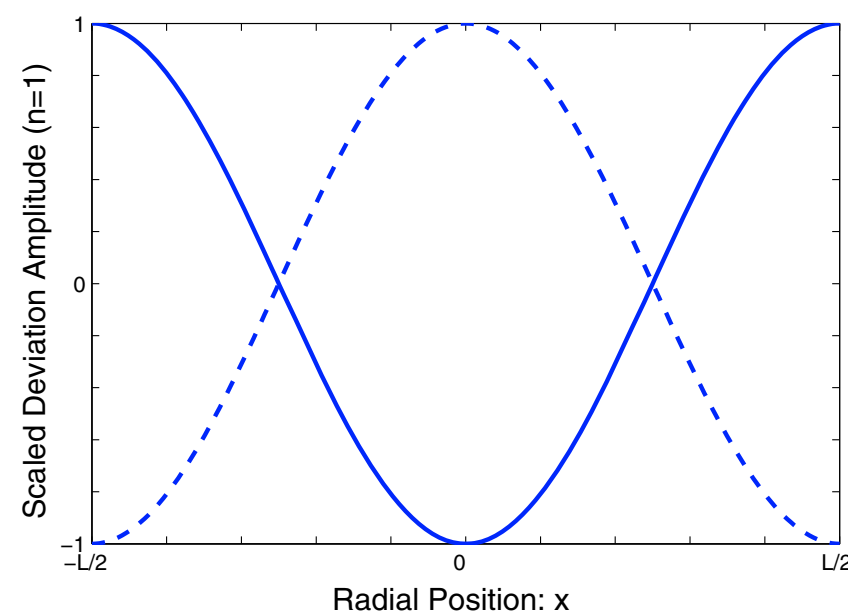

Fig. 2. The $n=1$ component of the shear profile (31). The solid line $q_{1}=1$ and the dashed line $q_{1}=-1$. For problems in which channel wall conditions are imposed the solid line corresponds to a stable deviation profile while the dashed line corresponds to an unstable profile. The situation is reversed when fixed-pressure boundary conditions are imposed.

which is very similar in form to (38) except that the solution in the domain is composed of even functions and the series includes the mode with $m=0$, which is the classical channel mode. The correction to the temporal response for all values of $m \neq 0$ is

$\sigma_{1 m}^{2}=q_{m}(-1)^{m} \Upsilon(m)$

which is the negative of the solution found in (41). As Fig. 2 indicates, this result says that shear profiles in a channel configuration that promote stability (instability) play a destabilizing (stabilizing) role for configurations with fixed-pressure boundary conditions.

However the $m=0$ mode is a special case which requires a separate analysis. In its simplest guise, the channel mode $\psi_{00}$ is a constant with respect to $x$. Because of the periodicity of $Q_{1}$ it means that $\left\langle Q_{1} \psi_{00}^{2}\right\rangle=0$ and the analysis advanced up until this point to evaluate the correction $\sigma_{1}$ shows that it will be zero at this order. Thus given this and the fact that $\kappa_{00}^{2}=0$ the solution must be expanded according to,

$$
\begin{aligned}
& \psi_{0}=\psi_{00}+\epsilon \psi_{10}+\epsilon^{2} \psi_{20}+\cdots \\
& \sigma^{2}=\sigma_{00}^{2}+\epsilon^{2} \sigma_{20}^{2}+\cdots \\
& \kappa_{0}^{2}=\epsilon^{2}\left(\frac{\partial \kappa_{0}^{2}}{\partial \sigma^{2}}\right)_{\sigma_{00}^{2}} \sigma_{20}^{2}
\end{aligned}
$$

$\left(\frac{\partial \kappa_{0}^{2}}{\partial \sigma^{2}}\right)=\frac{k^{2}}{\sigma_{00}^{2}+\omega_{a z}^{2}}\left(-\frac{\omega_{0}^{2}}{\sigma_{00}^{2}+\omega_{a z}^{2}}+\frac{8 \Omega_{0}^{2} \omega_{a z}^{2}}{\left(\sigma_{00}^{2}+\omega_{a z}^{2}\right)^{2}}\right)$,

where $\sigma_{00}^{2}$, i.e. the temporal response of the channel mode, is given in (29). The equations for the solution of $\psi$ are in successive order given by

$\partial_{x}^{2} \psi_{00}=0$

$\partial_{x}^{2} \psi_{10}=-\frac{2 \Omega_{0}^{2} k^{2} Q_{1}}{\sigma_{00}^{2}+\omega_{a z}^{2}} \psi_{00}$,

$\partial_{x}^{2} \psi_{20}=\left(\frac{\partial \kappa_{0}^{2}}{\partial \sigma^{2}}\right)_{\sigma_{00}^{2}} \sigma_{20}^{2} \psi_{00}-\frac{2 \Omega_{0}^{2} k^{2} Q_{1}}{\sigma_{00}^{2}+\omega_{a z}^{2}} \psi_{10}$. 
The first of these says that the lowest order solution which satisfies the boundary condition is a constant $\psi_{00}=A_{00}$. The next order down the solution is

$\psi_{10}=-\frac{2 \Omega_{0}^{2} k^{2}}{\sigma_{00}^{2}+\omega_{a z}^{2}} Q A_{00}$,

where

$$
\begin{aligned}
Q= & -\sum_{n=1}^{\infty} q_{n}\left(\frac{L}{2 n \pi}\right)^{2}\left[\sin \left(\frac{2 n \pi}{L} x+\theta_{n}\right)\right. \\
& \left.-\frac{2 n \pi}{L} x(-1)^{n} \cos \theta_{n}\right] .
\end{aligned}
$$

In other words the solution $Q$ is such that $\partial_{x}^{2} Q=Q_{1}$ which satisfies the boundary condition $\left.\partial_{x} Q\right|_{x= \pm L / 2}=0$. In order to generate a bounded solution for $\psi_{20}$ a solvability condition must be applied to the RHS of (50), which is obtained by re-expressing $\psi_{10}$ in terms of its solution as given in (51) and then integrating the equation from across the domain and setting the result to zero:

$\left(\frac{\partial \kappa_{0}^{2}}{\partial \sigma^{2}}\right)_{\sigma_{00}^{2}} \sigma_{20}^{2}=-\left(\frac{2 \Omega_{0}^{2} k^{2}}{\sigma_{00}^{2}+\omega_{a z}^{2}}\right)^{2}\left\langle Q_{1} Q\right\rangle A_{00}$.

However, given the relationship between $Q$ and $Q_{1}$ it follows after an integration by parts that in fact,

$$
\left(\frac{\partial \kappa_{0}^{2}}{\partial \sigma^{2}}\right)_{\sigma_{00}^{2}} \sigma_{20}^{2}=\left(\frac{2 \Omega_{0}^{2} k^{2}}{\sigma_{00}^{2}+\omega_{a z}^{2}}\right)^{2}\left\langle\left(\partial_{x} Q\right)^{2}\right\rangle .
$$

Given that for HMI modes

$\left(\frac{\partial \kappa_{0}^{2}}{\partial \sigma^{2}}\right)_{\sigma_{00}^{2}}>0$

the above solvability condition says something remarkable: that the channel mode is always destabilized no matter what $Q_{1}$ happens to be. By contrast, values of $q_{n}$ may be chosen so that each eigenmode of the system with non-trivial radial structure (i.e. $m>0$ ) may be stably influenced by the shear $Q_{1}$, yet, there is no such configuration of the disturbed shear flow that can act to stabilize a channel mode. This result, above all others, emphasizes the important uniqueness of these modes. Also worth noting is that while the corrections to the growth rates of all modes (except $m=0$ ) is proportional to $\epsilon$, the corresponding growth rate correction for the channel mode scales as $\epsilon^{2}$ : which means that though the channel mode is consistently destabilized by any periodic shear profile, its influence is markedly weaker.

\subsubsection{Periodic conditions}

Solutions of $\psi_{0 m}$ that are periodic on the domain $L$ are given by

$\psi_{0 m}=A_{m} \sin \left[\beta_{m}(x+L / 2)+\phi_{m}\right]$,

where $\beta_{m} \equiv 2 m \pi / L$ and $\phi_{m}$ is an arbitrary phase. The temporal response is given by $\sigma_{0 m}^{2}\left(\beta_{m}^{2}\right)$, as defined in (26). As in the previous section, if attention is first given to those modes where $m \neq 0$, then one finds that

$\sigma_{1 m}^{2}=q_{2 m} \frac{1}{2}(-1)^{m} \sin \left(\frac{\pi}{2}-2 \phi_{m}\right) \Upsilon(m)$.
As in the previous section, the correction $\sigma_{1 m}^{2}$ for the channel mode $(m=0)$ is zero and the first non-trivial temporal response comes at order $\epsilon^{2}$. The procedure is exactly the same as found in Sect. 5.2.2 and its correction $\sigma_{20}^{2}$ is

$$
\left(\frac{\partial \kappa_{0}^{2}}{\partial \sigma^{2}}\right)_{\sigma_{00}^{2}} \sigma_{20}^{2}=\left(\frac{2 \Omega_{0}^{2} k^{2}}{\sigma_{00}^{2}+\omega_{a z}^{2}}\right)^{2}\left\langle\left(\partial_{x} \tilde{Q}\right)^{2}\right\rangle,
$$

with

$\tilde{Q}=-\sum_{n=1}^{\infty} q_{n}\left(\frac{L}{2 n \pi}\right)^{2}\left[\sin \left(\frac{2 n \pi}{L} x+\frac{\pi}{2}\right)\right]$.

Imposing periodic boundary conditions also implies that there are values of the coefficients of the perturbed shear $Q_{1}$ which can be chosen to promote stability for all the modes of the system except for the channel mode. For example, for the $m=1$ mode and given its relative phase $\phi_{1}$ one can choose $q_{2}$ so that the RHS of (55) is negative. This can be done for all other modes of the system as well except for the $m=0$ channel mode, which is destabilized no matter what choice is made for the parameters of $Q_{1}$, i.e. the set $q_{n}$.

\subsection{Single shear defect on an infinite domain: localized disturbances}

A profile with a single shear defect located at $x=0$ is represented by the delta-function profile

$Q=Q_{0} L \delta(x)$

The corresponding departures from the mean Keplerian shear profile $U$ is given by

$U=\left\{\begin{array}{rr}-Q_{0} \Omega_{0} L / 2, & x<0 \\ Q_{0} \Omega_{0} L / 2, & x>0 .\end{array}\right.$

If $\psi_{ \pm}$represents the solution of (15) respectively for $x>0$ and $x<0$, then solutions which decay as $x \rightarrow \pm \infty$ are

$\psi_{ \pm}=A_{ \pm} \mathrm{e}^{\mp\left|\kappa_{0}\right| x}$

where $\kappa_{0}$ is as given in (22) and provided that $\kappa_{0}^{2}>0$. If the latter condition is not met, then there are no localized normal modes allowed. The coefficients of the streamfunctions in both regions are equal in order that they be continuous across $x=0$, thus $A_{+}=A_{-}=A$. Integrating (15) in an infinitesimal region around $x=0$, and given the defect (58), imposes a jump condition for the derivatives of $\psi$ approaching either side of the defect,

$\left.\partial_{x} \psi\right|_{0^{+}}-\left.\partial_{x} \psi\right|_{0^{-}}=-\frac{2 \Omega_{0}^{2} k^{2}}{\sigma^{2}+\omega_{a z}^{2}} Q_{0} L A$.

Putting in the solutions for the respective domains gives the quantization criterion

$\left|\kappa_{0}\right|=\frac{\Omega_{0}^{2} k^{2}}{\sigma^{2}+\omega_{a z}^{2}} Q_{0} L$.

Solutions of the above equation are constrained to two possibilities: (i) if $Q_{0}<0$, then $\sigma^{2}+\omega_{a z}^{2}<0$ for a normal mode solution to exist while (ii) if $Q_{0}>0$, then it must be that $\sigma^{2}+\omega_{a z}^{2}>0$ for there to be a normal mode. The first of these cases says that normal modes are allowed only for those values of $\sigma^{2}$ which are negative which are associated with the HI modes. The second of these cases are associated with the HMI modes of which the MRI 
is a possibility. Thus it is an interesting result that the MRI is a permitted localized normal mode disturbance only if the shear defect is positive. If the shear defect is negative (so that there is an effective reduction of the shear inside the region), then there are no normal modes predicted.

The solution of (62) is obtained by first taking the square of both sides of (62) yielding,

$1-\frac{4 \Omega_{0}^{2} \omega_{a z}^{2}}{\left(\sigma^{2}+\omega_{a z}^{2}\right)^{2}}+\frac{\omega_{0}^{2}}{\sigma^{2}+\omega_{a z}^{2}}=\frac{\Omega_{0}^{4} k^{2}}{\left(\sigma^{2}+\omega_{a z}^{2}\right)^{2}} Q_{0}^{2} L^{2}$.

However one must be careful in interpreting the solutions of this simplified equation. Naively solving (63) will yield twice as many solutions than are allowed. This overcounting is corrected by requiring that only those solutions of (63) that satisfy

$Q_{0} /\left(\sigma^{2}+\omega_{a z}^{2}\right)>0$,

are permitted or else (62) cannot be satisfied. Solutions of (62) are,

$\sigma^{2}+\omega_{a z}^{2}=\frac{1}{2}\left[-\omega_{0}^{2} \pm \sqrt{\omega_{0}^{4}+4\left(Q_{0}^{2} k^{2} L^{2} \Omega_{0}^{4}+4 \Omega_{0}^{2} \omega_{a z}^{2}\right)}\right]$.

Because the term inside the radical sign is always greater than zero for $q_{0}>0$, the " + " branch of the above expression is always greater than zero while the "-" branch is always less than zero. Thus the "+" branch is the permitted solution for $Q_{0}>0$ while the "-" branch is the allowed one for $Q_{0}<0$. Incorporating this formally yields the expression

$\left.\sigma^{2}+\omega_{a z}^{2}=\frac{1}{2}\left[-\omega_{0}^{2}+\operatorname{sgn}\left(Q_{0}\right) \sqrt{\omega_{0}^{4}+4\left(Q_{0}^{2} k^{2} L^{2} \Omega_{0}^{4}+4 \Omega_{0}^{2} \omega_{a z}^{2}\right.}\right)\right]$.

Rewriting the terms appearing in the interior of the radical sign leads to finally,

$$
\begin{aligned}
\sigma^{2}= & -\left(\omega_{a z}^{2}+\frac{\omega_{0}^{2}}{2}\right)+\operatorname{sgn}\left(Q_{0}\right)\left[\left(\omega_{a z}^{2}+\frac{\omega_{0}^{2}}{2}\right)^{2}\right. \\
& \left.-\omega_{a z}^{2}\left(\omega_{a z}^{2}-2 \Omega_{0}^{2} q_{0}\right)+\Omega_{0}^{4}(k L)^{2} Q_{0}^{2}\right]^{1 / 2},
\end{aligned}
$$

which is similar in content to the classical channel mode dispersion relation (29) except for the fact that at any one time there exists only one branch of modes as a possible normal mode solution. The MRI mode only manifests as a normal mode when $Q_{0}>0$ and its growth rate is enhanced by the defect.

\subsection{Symmetric shear step on an infinite domain: localized disturbances}

A finite version of the shear profile evaluated in the previous section is,

$Q=\left\{\begin{aligned} 0, & x<-\frac{L}{2} \\ Q_{0}, & -\frac{L}{2}<x<\frac{L}{2} \\ 0, & x>\frac{L}{2} .\end{aligned}\right.$

This profile for $Q$ has a top-hat structure and this descriptor will be used interchangeably with the expression symmetric shear step. Evidentally the value of the integral, $\int_{-\infty}^{\infty} Q \mathrm{~d} x=Q_{0} L$, is the same for both forms of $Q$ given in (58) and (65). Solutions to (15) must be developed separately in the three regions and appropriately matched across the boundaries separating the regions. Because $Q$ is bounded it will suffice to match both the streamfunctions and their first derivatives across the domain. Thus, for $x<-L / 2$ and $x>L / 2(15)$ is

$\partial_{x}^{2} \psi-\kappa_{0}^{2} \psi=0$.

The solutions in the region where $x<-L / 2$ is given by

$\psi_{-}=A_{-} \mathrm{e}^{\left|\kappa_{0}\right|(x+L / 2)}$,

while for $x>L / 2$

$\psi_{-}=A_{+} \mathrm{e}^{-\left|\kappa_{0}\right|(x-L / 2)}$

together with the constraint that $\kappa_{0}^{2}>0$. In the region $-L / 2<$ $x<L / 2(15)$ is

$\partial_{x}^{2} \psi-\left(\kappa_{0}^{2}-\frac{2 \Omega_{0}^{2} Q_{0} k^{2}}{\sigma^{2}+\omega_{a z}^{2}}\right) \psi=0$,

which has the two linearly independent solutions ${ }^{7}$

$\psi_{0}=A_{0} \cosh [\kappa x]+B_{0} \frac{\sinh [\kappa x]}{\kappa}$,

in which

$\kappa^{2}\left(\kappa_{0}\right) \equiv \kappa_{0}^{2}-\frac{2 \Omega_{0}^{2} Q_{0} k^{2}}{\Sigma^{( \pm)}\left(\kappa_{0}, \omega_{0}\right)}$.

The functional form

$\sigma^{2}+\omega_{a z}^{2}=\Sigma^{( \pm)}\left(\kappa_{0}, \omega_{0}\right)$

in which

$\Sigma^{( \pm)}\left(\kappa_{0}, \omega_{0}^{2}\right) \equiv$

$$
\frac{1}{2\left(1-\frac{k_{0}^{2}}{k^{2}}\right)}\left[-\omega_{0}^{2} \pm\left[\omega_{0}^{4}+16 \Omega_{0}^{2} \omega_{a z}^{2}\left(1-\frac{\kappa_{0}^{2}}{k^{2}}\right)\right]^{1 / 2}\right],
$$

is introduced in order to make the following discussion more transparent. The \pm designation references the HMI or HI modes respectively. It is also worth noting that given the constraint that $\sigma^{2}$ must be real, that there is an absolute maximum value allowed for $\kappa_{0}^{2}$ and this is dictated by the requirement that the terms subject to the square root operator must remain greater than zero or else the value of $\sigma^{2}$ will turn out complex. In other words, given the reality of $\sigma^{2}$ one does not expect solutions of $\kappa_{0}^{2}$ to exceed $\kappa_{\max }^{2}$ where

$\kappa_{\max }^{2}=k^{2}\left(\frac{\omega_{0}^{4}}{16 \Omega_{0}^{2} \omega_{a z}^{2}}+1\right)$.

In all of the numerical solutions calculated below it is found that, indeed, all the solutions determined satisfy $\kappa_{0}^{2}<\kappa_{\max }^{2}$.

Imposing the conditions that the streamfunction and its first derivative match across the two boundaries $x= \pm L / 2$ reveals that normal mode solutions exist provided the following condition is satisfied

$\left[\tanh \left(\kappa \frac{L}{2}\right)+\frac{\kappa}{\left|\kappa_{0}\right|}\right]\left[\operatorname{coth}\left(\kappa \frac{L}{2}\right)+\frac{\kappa}{\left|\kappa_{0}\right|}\right]=0$.

The terms in the first bracket represent a quantization condition for odd-parity modes, which is to say that in the dimpled region

\footnotetext{
7 The second of the solutions appearing in (70) is written in the form displayed in order to make sure that both linearly independent solutions are represented even in the event $\kappa=0$ (Friedman 1990).
} 
$\psi_{0} \sim \sinh [\kappa x] / \kappa$, while the second represents even-parity modes where similarly $\psi_{0} \sim \cosh [\kappa x]$ in the same region.

Attention is first given to the even-parity mode in which the quantization condition,

$\operatorname{coth}\left(\kappa \frac{L}{2}\right)=-\frac{\kappa}{\left|\kappa_{0}\right|}$

must be satisfied for some value of $\kappa_{0}$. If $\kappa$ were real and greater than zero, then the LHS of the above relationship is always positive. Since $\kappa_{0}^{2}$ is real and positive, if the modes are to be localized, then the RHS of this expression is always less than zero. In this case it means that no solution exists for $\kappa$ real. On the other hand solutions do exist if $\kappa^{2} \leq 0$. Rewriting $\kappa=i \widetilde{\kappa}$ then the quantization condition above becomes

$\cot [\tilde{\kappa} L / 2]=\frac{\tilde{\kappa}}{\left|\kappa_{0}\right|}$,

now with the constraint for the existence of localized normal modes is re-expressed as

$\tilde{\kappa}^{2}\left(\kappa_{0}\right) \equiv \frac{2 \Omega_{0}^{2} Q_{0} k^{2}}{\Sigma^{( \pm)}\left(\kappa_{0}, \omega_{0}\right)}-\kappa_{0}^{2}>0$.

The strategy for obtaining a solution is as follows: find the values of $\kappa_{0}$ which solve (76), subject to the constraint given in (77), and then given the solution $\kappa_{0}$ find $\sigma^{2}$ through the relationship (72). The same procedure will be implemented for the even-parity solution below as well. Solutions of the quantization condition will be sought graphically in the range of values for $\kappa_{0} \in\left(0, \kappa_{\max }\right)$. Inspection shows that the constraint (77) is violated if $Q_{0}<0$ and $\Sigma^{( \pm)}>0$, or if $Q_{0}>0$ and $\Sigma^{( \pm)}<0$. Since $\Sigma^{(+)}>0$ it means that there are no HMI normal modes as allowable solutions if $Q_{0}<0$. HI normal modes are associated with the $\Sigma^{(-)}$branch of solutions and since this is less than zero except for very special circumstances, one can generally expect HI normal modes for $Q_{0}<0$ and for them to be stable. The aforementioned special circumstance occurs if there are solutions to (76) in which $\kappa_{0}>k$ since, in that case, $\Sigma^{(-)}>0$ and $\mathrm{HI}$ modes may exist as well for $Q_{0}>0$.

The even-parity mode limits to the single-defect configuration discussed in Sect. 5.3 for small values of the parameter $L$. Analysis of (76) in this limit shows that,

$\frac{2}{\tilde{\kappa} L} \approx \frac{\tilde{\kappa}}{\left|\kappa_{0}\right|}$

which, after restoring the definition of $\tilde{\kappa}$ and some rearranging, becomes

$\left|\kappa_{0}\right|=\frac{L}{2}\left(\frac{2 \Omega_{0}^{2} Q_{0} k^{2}}{\sigma^{2}+\omega_{a z}^{2}}-\kappa_{0}^{2}\right)$,

where the definition of $\Sigma^{( \pm)}$has been restored. For values of $Q_{0}$ such that $\Omega_{0}^{2} Q_{0} \gg\left(\sigma^{2}+\omega_{a z}^{2}\right) \kappa_{0}^{2} / k^{2}$ the above expression reduces to

$\left|\kappa_{0}\right|=\frac{\Omega_{0}^{2} Q_{0} L k^{2}}{\sigma^{2}+\omega_{a z}^{2}}$,

which is the quantization condition for the single-defect problem on the infinite domain, (62). The range of values for $Q_{0}$ and $L$ for which this limiting form agrees with the actual result of the quantization condition is shown in Fig. 3, where $\omega_{a z}^{2}$ is set to the value $2 \Omega_{0}^{2} q_{0}$ in order to compare the temporal response against conditions in which the channel mode is marginal in the classical (a) Temporal Response, $Q_{0}=0.6$ (HMI Mode)

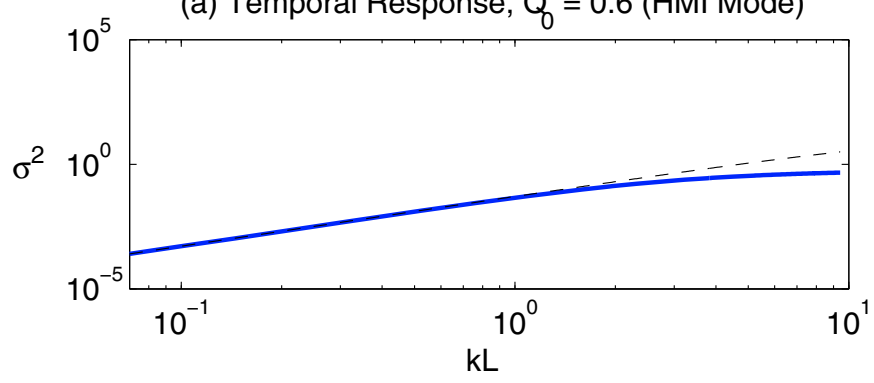

(b) Temporal Response, $Q_{0}=-0.6$ (HI Mode)

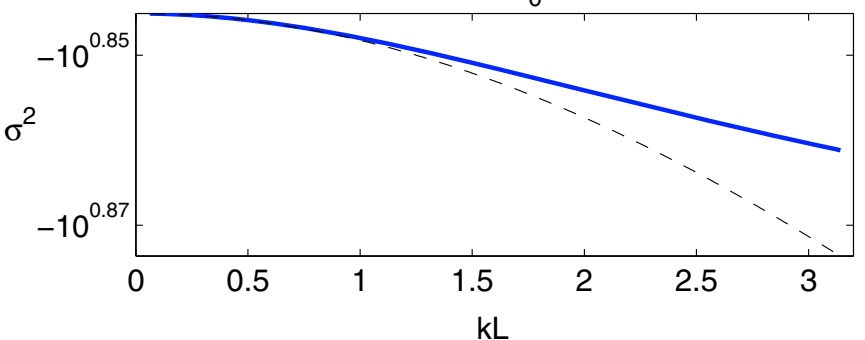

Fig. 3. The temporal response resulting from the quantization condition (76) as a function of $k L$ (solid line) compared to the quantization condition (62) for defect profile (dashed line). For both plots $\omega_{a z}^{2}=2 \Omega_{0}^{2} q_{0}$ with $q_{0}=3 / 2$. a) For $Q_{0}>0$ only HMI normal modes are permitted. The agreement between the two profiles severely breaks down for $k L$ exceeding 2. b) For $Q_{0}<0$ only $\mathrm{HI}$ normal modes are allowed. The agreement between defect theory and step profile is better for HI modes.

theory, see (28). The main result is that the temporal response of the defect profile of the previous section closely resembles the temporal response of this step profile here in the limit where $k L$ is small less than one. The limiting form represented by the defect profile becomes a poor representation of the top-hat profile when $k L$ exceeds order 1 values, however, the agreement tends to be much better for the HI.

Additionally, for small values of $k L$ there is always at least one normal mode expected, whether it be the HI or HMI mode. However, as the horizontal length of the symmetric step profile increases, the number of permitted normal modes increases as well (Fig. 4). Similar behavior is predicted for modes which are localized in the vertical direction of the disk (Liverts \& Mond 2009). As the number of permitted normal modes increases their $\kappa_{0}$ values will cluster (countably as $k L \rightarrow \infty$ ) around the maximum value

$$
\begin{aligned}
& \left(\kappa_{0}^{2}\right)_{\max }= \\
& \frac{Q_{0}}{4 \omega_{a z}^{2}}\left(\omega_{0}^{2}-2 Q_{0} \Omega_{0}^{2}+\sqrt{\left(\omega_{0}^{2}-2 Q_{0} \Omega_{0}^{2}\right)^{2}+16 \Omega_{0}^{2} \omega_{a z}^{2}}\right) .
\end{aligned}
$$

Odd-parity modes are interesting since they have no analogues in the single defect profile studied in the previous section. Examining the quantization condition for it,

$\tanh \left(\kappa \frac{L}{2}\right)+\frac{\kappa}{\left|\kappa_{0}\right|}=0$

shows that there are no real solutions of $\kappa$ that satisfy the constraint. Utilizing the expression $\kappa=i \tilde{\kappa}$, as used previously, the quantization condition takes the form

$\tan [\tilde{\kappa} L / 2]=-\frac{\tilde{\kappa}}{\left|\kappa_{0}\right|}$. 


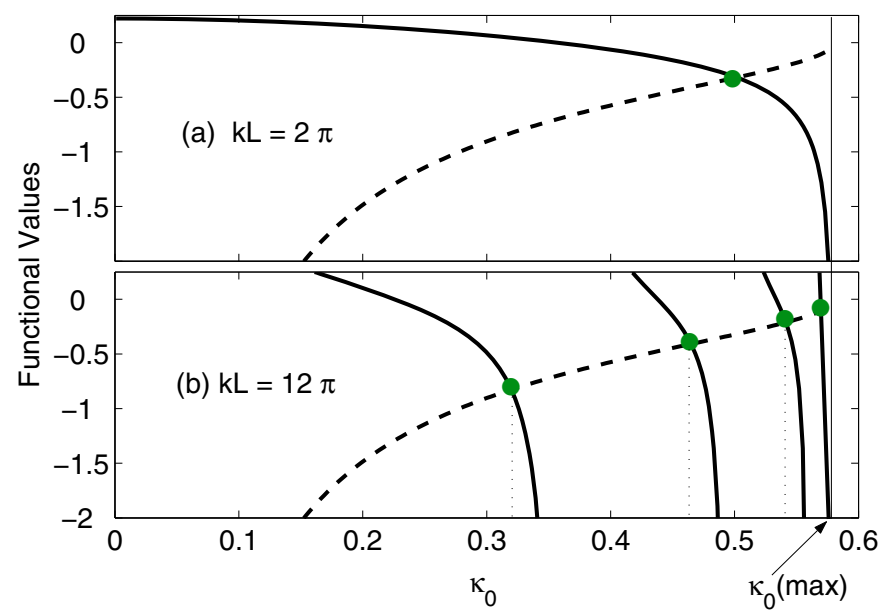

Fig. 4. Plot showing the increase of allowed normal modes with domain size $L$. In all plots $\omega_{a z}^{2}=2 \Omega_{0}^{2} q_{0}, q_{0}=3 / 2$ and $Q_{0}=0.6$ (HMI mode permitted). The dashed line is $\tilde{\kappa} /\left|\kappa_{0}\right|$ and solid line is $\cot \tilde{\kappa} L / 2$. Panel a): $k L=2 \pi$ corresponds to one normal mode at $\kappa_{0}=0.50$. Panel b): $k L=12 \pi$ supporting four normal modes $\kappa_{0}=0.32,0.465,0.54,0.56$. All modes correspond to values of $\sigma^{2}>0 . \kappa_{0}(\max )=0.576$.

Inspection of the condition (79) shows that for there to exist a normal mode $\tilde{\kappa}$ must be greater than $\pi / L$ as the functional form $\tan [\tilde{\kappa} L / 2] / \tilde{\kappa}$ is positive for values of $\tilde{\kappa} \in(0, \pi / L)$. This places a constraint upon the minimum value of $L$ that permits this mode to exist and this is obtained by solving

$\tilde{\kappa}=\frac{\pi}{k L}$,

and, using the definition for $\kappa$ found in (71) one finds that the minimum value for $L$ must satisfy,

$k L_{\min }=\left[\frac{\omega_{0}^{2} \pi^{2}}{4 Q_{0}}\left(\operatorname{sgn}\left(Q_{0}\right) \sqrt{1+\frac{16 \Omega_{0}^{2} \omega_{a z}^{2}}{\omega_{0}^{4}}}-1\right)\right]^{1 / 2}$.

Note that the inclusion of the expression $\operatorname{sgn}\left(Q_{0}\right)$ reflects how $\mathrm{HMI} / \mathrm{HI}$ modes associate with the sign of $Q_{0}$ so that the expression appearing above encapsulates both possibilities. Odd-parity modes bifurcate into existence for $L \rightarrow \infty$ as $Q_{0}$ grows from zero and therefore it means that these modes are not expected to play a role for values of the defect which are small as this would entail very large values of the top-hat region. The temporal response of these odd-parity modes are otherwise similar in most every respect to their even-parity counterparts and, as such, no further analysis of their properties is pursued here.

It should be noted that as in the previous section unstable localized MRI normal modes (HMI-modes) can exist only if the step function in the shear is greater than zero. If the step is negative (indicating a region of weakened shear), then there are no HMI types of localized normal modes admitted by the configuration and, hence, there is no possibility of normal mode unstable disturbances.

\subsection{Single shear defect on a periodic domain}

Next a shear defect of the form

$Q=-Q_{0}+Q_{0} L \delta(x)$,

is considered on the periodic domain $-L / 2 \leq x \leq L / 2$. This form is very similar to the one considered in Sect. 5.3 except the integral of the shear over the periodic domain is zero, i.e. $\langle Q\rangle=$ 0 . The effects of shear defects with zero mean may be examined. The governing equation for $\psi$ is

$\partial_{x}^{2} \psi-\kappa_{00}^{2} \psi=-\frac{2 \Omega_{0}^{2} k^{2} Q_{0} L \delta(x)}{\sigma^{2}+\omega_{a z}^{2}}$,

where $\kappa_{00}$ is given by

$\kappa_{00}^{2}=k^{2}\left(1+\frac{4 \Omega_{0}^{2} \sigma^{2}}{\left(\sigma^{2}+\omega_{a z}^{2}\right)^{2}}-\frac{2 \Omega_{0}^{2}\left(q_{0}-Q_{0}\right)}{\sigma^{2}+\omega_{a z}^{2}}\right)$.

Similar to what was done in the previous sections, one may express $\sigma$ in terms of $\kappa_{00}$ via the expression $\Sigma^{( \pm)}$and, for the sake of completeness, this is written out explicitly,

$$
\begin{aligned}
& \sigma^{2}+\omega_{a z}^{2}=\Sigma^{( \pm)}\left(\kappa_{00}, \omega_{00}^{2}\right) \equiv \\
& \frac{1}{2\left(1-\frac{\kappa_{00}^{2}}{k^{2}}\right)}\left[-\omega_{00}^{2} \pm\left[\omega_{00}^{4}+16 \Omega_{0}^{2} \omega_{a z}^{2}\left(1-\frac{\kappa_{00}^{2}}{k^{2}}\right)\right]^{1 / 2}\right]
\end{aligned}
$$

in which the shear modified epicyclic frequency is defined as $\omega_{00}^{2} \equiv 2 \Omega_{0}^{2}\left(2-q_{0}+Q_{0}\right)$. Solutions to (83) must be developed separately for either side of $x=0$ and matched. Solutions for the streamfunction in which $\psi(-L / 2)=\psi(L / 2)$ and $\left.\partial_{x} \psi\right|_{-L / 2}=$ $\left.\partial_{x} \psi\right|_{L / 2}$ (i.e. that they are periodic) are given by

$\psi_{ \pm}=A \cosh \left[\kappa_{00}\left(x \mp \frac{L}{2}\right)\right]$

where $\psi_{ \pm}$is the solution for the region $x \in(0, \pm L / 2)$. Continuity of the stream function is ensured by construction of the form of the solution in (86). The streamfunction must show a jump in its first derivative following the same arguments found in Sect. 5.3. Integrating (83) in a small region around $x=0$ results in the same condition for the jump in $\partial_{x} \psi$ found in (61). Putting in the forms for $\psi_{ \pm}$found in (86) leads to the quantization condition

$\kappa_{00} \tanh \left[\kappa_{00} \frac{L}{2}\right]=\frac{\Omega_{0}^{2} k^{2} Q_{0} L}{\Sigma^{( \pm)}\left(\kappa_{00}, \omega_{00}^{2}\right)}=\mathcal{F}^{( \pm)}\left(\kappa_{00}, \omega_{00}^{2}\right)$.

It should be kept in mind that the quantities $\mathcal{F}^{( \pm)}$as appearing on the RHS of (87) are different functions of $\kappa_{00}$ depending upon whether one is considering $\mathrm{HMI} / \mathrm{HI}( \pm)$ modes, respectively. Thus when solutions of the quantization condition are sought, separate attention will be given depending upon which type of mode is of interest. Furthermore, consideration of the solutions to (87) must be done by restricting attention to cases where $\kappa_{00}$ is either real or imaginary. Additionally from previous arguments showing that $\sigma^{2}$ must be real, there will be a maximum real value possible for $\kappa_{00}$

$\kappa_{\max }^{2}\left(\omega_{00}^{2}\right)=k^{2}\left(\frac{\omega_{00}^{4}}{16 \Omega_{0}^{2} \omega_{a z}^{2}}+1\right)$

Values of $\kappa_{00}$ which are greater that $\kappa_{\max }\left(\omega_{00}^{2}\right)$ would lead to complex values of $\sigma^{2}$ as an inspection of (85) shows that if $\kappa_{00}$ does exceed this maximum value, then the term inside the square-root operator becomes negative which would lead to a complex value of $\sigma^{2}$. This restriction upon the value of $\kappa_{00}$ helps in finding solutions for it.

The limit where $Q_{0} \rightarrow 0$ recovers the classical limits discussed in Sect. 5.1. In that case channel modes, i.e. those modes 
O. M. Umurhan: Speculation on low magnetic Prandtl number disk velocity profiles

(a) $Q_{0}=0.1$

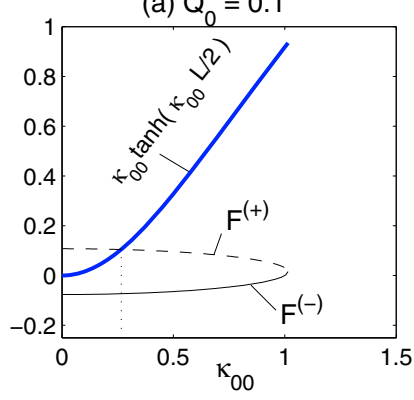

(c) $Q_{0}=2.0$

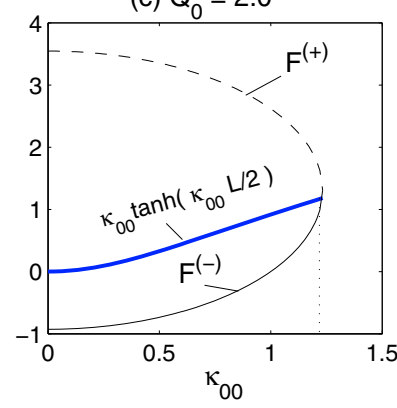

(b) $Q_{0}=1.5$

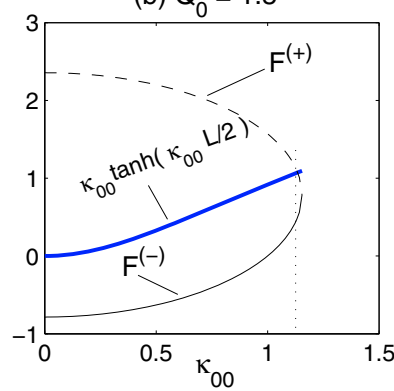

(d) $Q_{0}=3.5$

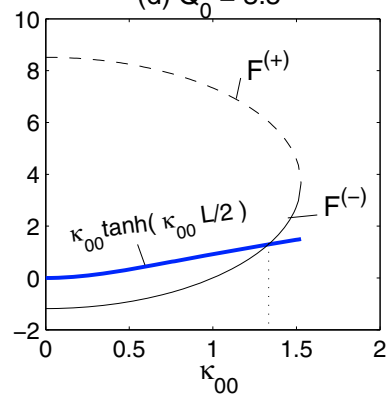

Fig. 5. Graphical solutions for the quantization condition (87). Attention restricted to real values of $\kappa_{00}$ marking the mode which becomes the classical channel mode in the limit $Q_{0} \rightarrow 0$. Each panel depicts the three functions $\kappa_{00} \tanh \kappa_{00} L / 2$ (thick line), and $\mathcal{F}^{( \pm)}$ (dashed/thin lines). In all plots $k L=\pi$ and $\omega_{a z}^{2}=2 \Omega_{0}^{2} q_{0}$. Panels a) and b) show that HMI modes are admitted as the solid curve crosses the $\mathcal{F}^{(+)}$line while for the latter two it crosses the line corresponding to $\mathcal{F}^{(-)}$meaning that $\mathrm{HI}$ modes are selected. The solution $\kappa_{00}$ and temporal response for each: a) $Q_{0}=0.1, \kappa_{00}=0.27, \sigma^{2}=0.005$, b) $Q_{0}=0.5, \kappa_{00}=0.65, \sigma^{2}=0.14$, c) $Q_{0}=2.0, \kappa_{00}=1.33, \sigma^{2}=3.36$ and d) $Q_{0}=3.5, \kappa_{00}=1.37, \sigma^{2}=6.73$. Notice how the channel mode goes from being an unstable HMI mode to an unstable HI mode as $Q_{0}$ is increased.

for which $\kappa_{0}=0$, are the most unstable. In the current setting this corresponds to $\kappa_{00}=0$ when $Q_{0}=0$. Additionally, for $\omega_{a z}^{2}=2 \Omega_{0}^{2} q_{0}$ the channel mode is exactly marginal for $Q_{0}=0$, viz. the arguments leading to (28). This value for $\omega_{a z}^{2}$ will be assumed so that a controlled analysis may be carried out in which one can track how varying $Q_{0}$ affects the stability characteristics of an otherwise marginal channel mode.

Figures 5, 6 show the graphical solutions of (87) with some details of the results. The main result is that the mode that is identified with the classical channel mode in the ideal limit always exhibits exponential temporal growth no matter what amplitude the shear defect takes. Figure 7 shows that the temporal response of the classical channel mode is maximal for $Q_{0}=0$ and that as $Q_{0}$ moves away from zero instability is predicted. The mode exhibits an exponentially decaying spatial character $\left(\kappa_{00}^{2}>0\right)$ for $Q_{0}>0$, while it is weakly oscillatory $\left(\kappa_{00}^{2}<0\right)$ for $Q_{0}<0$. This general trend persists for a wide range of $k L$ values. As such, the results depicted in these figures can be taken to be qualitatively representative. The figures also indicate that the unstable HMI mode can also turn into an unstable HI mode if the amplitude of $Q_{0}$ becomes large enough.

\subsection{Single shear defect on finite domain: channel boundary conditions}

This section is concerned with the response of disturbances to a $Q$ configuration that is exactly like that considered in Sect. 5.5,

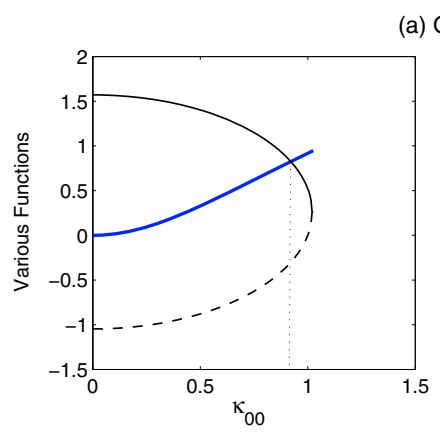

(a) $Q_{0}=-1.0$

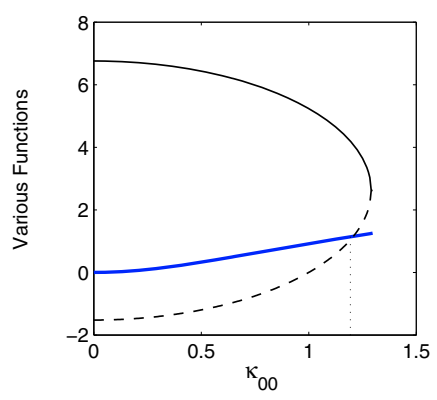

(b) $Q_{0}=-2.5$
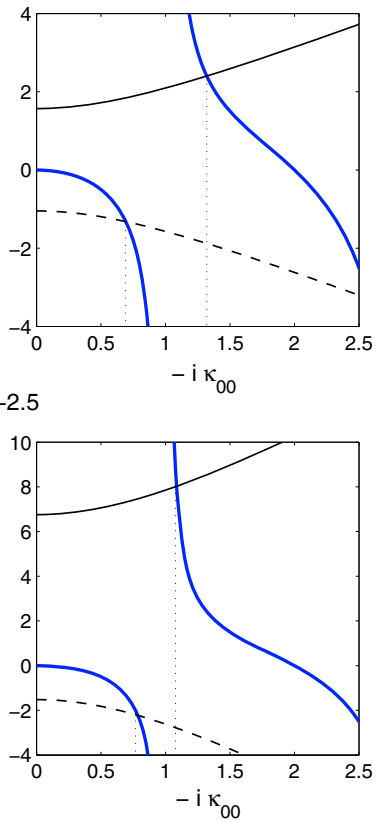

Fig. 6. Similar to Fig. 5 except negative values of $Q_{0}$ are investigated. In all plots $k L=\pi$ and $\omega_{a z}^{2}=2 \Omega_{0}^{2} q_{0}$ and $q_{0}=3 / 2$. Panel a): the left plot shows that the channel mode admitted is a stable HI mode with $\kappa_{00}=0.92$ and $\sigma^{2}=-5.30$. The right plot in panel a) shows the first two overtone modes. The first HMI mode is unstable with $\kappa_{00}=0.69 i$ and $\sigma^{2}=0.88$ while the next HI mode admitted has $\kappa_{00}=1.32 i$ and $\sigma^{2}=-2.88$. Note that in these cases $\kappa_{00}$ is imaginary. Panel b): stable HMI mode with $\kappa_{00}=1.21$ and $\sigma^{2}=-8.30$. For the first two overtones, the HMI mode is unstable with $\kappa_{00}=0.78 i$ and $\sigma^{2}=2.02$ while for the HI mode, $\kappa_{00}=1.08 i$ and $\sigma^{2}=-2.47$. For all values of $Q_{0}<0$ there is at least one unstable HMI mode with imaginary $\kappa_{00}$.

where the difference now is that the $\psi$ disturbances are set to zero at the boundaries $x= \pm L / 2$. This is equivalent to enforcing zero normal velocity at the boundaries. Thence, $\psi$ in this case is

$\psi_{ \pm}=\mp A \frac{\sinh \left[\kappa_{00}\left(x \mp \frac{L}{2}\right)\right]}{\sinh \kappa_{00} L / 2}$,

where just as in the previous section, $\psi_{ \pm}$is the solution for the region $x \in(0, \pm L / 2)$. Evaluating the jump condition at $x=0$ leads to an analogous quantization condition like that in (87), namely,

$\kappa_{00} \operatorname{coth}\left[\kappa_{00} \frac{L}{2}\right]=\frac{\Omega_{0}^{2} k^{2} Q_{0} L}{\sigma^{2}+\omega_{a z}^{2}}=\frac{\Omega_{0}^{2} k^{2} Q_{0} L}{\Sigma^{( \pm)}\left(\kappa_{00}, \omega_{00}^{2}\right)}$,

in which $\Sigma^{( \pm)}\left(\kappa_{00}, \omega_{00}^{2}\right)$ and $\kappa_{00}$ and $\omega_{00}^{2}$ are exactly as they are found in Sect. 5.5. It can be seen here, as before, that there there are never real solutions of $\kappa_{00}$ for HMI modes if $Q_{0}<0$ and, likewise, no such solutions for HI modes if $Q_{0}>0$.

In Fig. 8 the stabilizing behavior of the shear defect on the HMI modes is depicted for the most unstable mode of the system. The results from the weak shear limit discussed in Sect. 5.2.1 are used as a reference point to help interpret the following. From the classical theory discussed in that section it was established that the most unstable normal mode allowed is the one in which $m=1$, in turn implying $\gamma_{1}=\pi / L$. Reference to (27) shows that this mode is marginally stable when

$\omega_{a z}^{2}=2 \Omega_{0}^{2} q_{0} \frac{k^{2} L^{2}}{k^{2} L^{2}+\pi^{2}}$. 


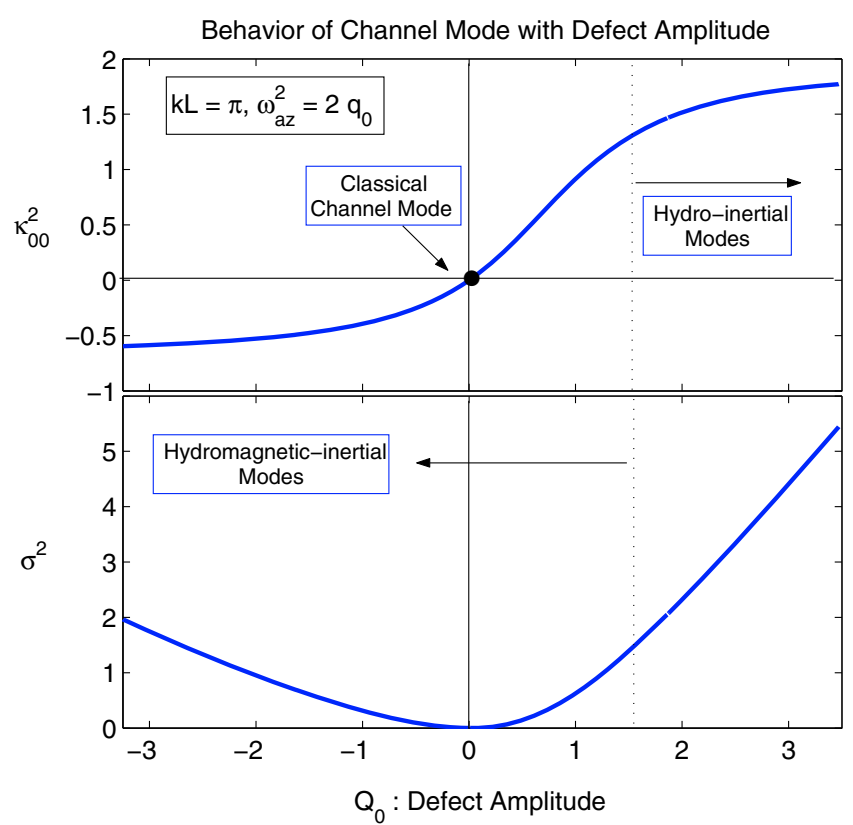

Fig. 7. Behavior of the classical channel mode as a function of defect amplitude $Q_{0}$ for $k L=\pi$ and $\omega_{a z}^{2}=2 \Omega_{0}^{2} q_{0}, q_{0}=3 / 2$, corresponding to a marginal $\left(\sigma^{2}=0\right)$ channel mode at $Q_{0}=0$. Top panel depicts $\kappa_{00}^{2}$ as a function of $Q_{0}$ in which the classic channel mode has $\kappa_{00}^{2}=0$. Bottom panel shows the temporal response which predicts exponential growth/decay for all values $Q_{0} \neq 0$. The channel mode goes from being a HMI mode to a HI mode at $Q_{0} \approx 1.87$.

(This is obtained by replacing $\beta_{n}$ with $\gamma_{1}$ in that expression.) The response of the modes for three different values of $k L$ are depicted. With the above choice for $\omega_{a z}^{2}$ all modes are marginal at $Q_{0}=0$. For the range of parameters investigated and shown in the figure, $\kappa_{00}$ must be imaginary in order for the quantization condition to be satisfied. Thus the values for $\kappa_{00}$ are written as $i \tilde{\kappa}_{00}$. Inspection of the results show that stability is promoted only for values of $Q_{0}$ which are negative. The range of values of $Q_{0}$ for which stability is predicted decreases for larger domain sizes, i.e. as $L$ is made larger the window in $Q_{0}$ for which disturbances are stable decreases. For those ranges in $Q_{0}$ in which stability is predicted, e.g. for $Q_{0} \in(-0.5,0)$ for $k L=2 \pi$ with $\omega_{a z}^{2}$ given in (91), the temporal response of the other overtones was checked and all were found to have $\sigma^{2}<0$.

\section{Discussion}

This study is devoted to examining the axisymmetric inviscid linear response of a shearing sheet environment threaded by a constant vertical magnetic field for an array of different shear profiles. The motivation for considering this setup are restated from the Introduction:

1. Numerical experiments of low magnetic Prandtl number flows appropriate for cold disk environments are outside current computational reach.

2. Current numerical experiments seem to show the trend that as $P_{\mathrm{m}}$ is lowered so is the corresponding angular momentum transport. The reasons for this is not yet clear.

3. Theoretical analysis of laboratory setups evaluating the response of the MRI subject to a vertical background field find that transport is reduced with $P_{\mathrm{m}}$. Furthermore this reduction emerges through the modification of the basic shear profile (e.g. Taylor-Couette) inside the experimental cavity which,

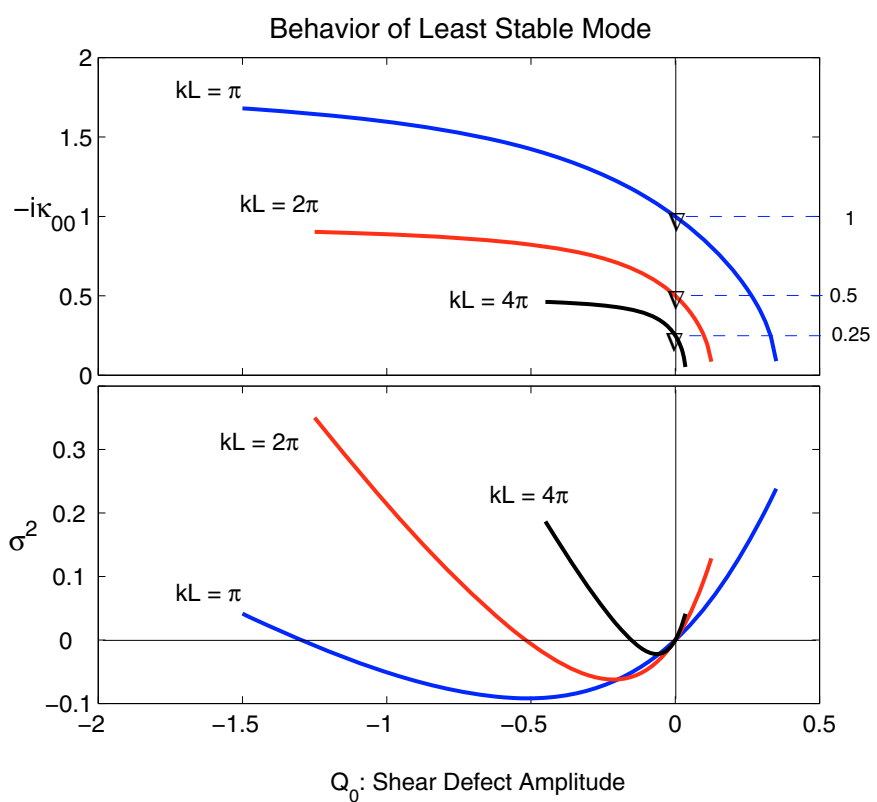

Fig. 8. The behavior of the first unstable (HMI) mode for the problem with channel walls $\left(q_{0}=3 / 2\right)$. The values of $i \tilde{\kappa}_{00}=\kappa_{00}$ and the corresponding temporal response $\sigma^{2}$ are given as a function of the defect amplitude $Q_{0}$ for three values of the parameter $k L$. The top panel shows $\tilde{\kappa}_{00}$. The bottom panel shows the temporal response. The value of $\omega_{a z}^{2}$ for each curve is set so that the temporal response is marginal at $Q_{0}=0-$ details of this is described in the text. The value of $\tilde{\kappa}_{00}$ for $Q_{0}=0$ are shown by the $\nabla$ symbol in the top panel.

in turn, is driven into place by the MRI itself. The final condition is a stable pattern state.

4. In the final pattern state reached by the model laboratory system the modified shear profile's amplitude is independent of $P_{\mathrm{m}}$. This is not true of the remaining fluid and magnetic quantities which scale as $P_{\mathrm{m}}^{\lambda}$ with typical values $\lambda \approx 1 / 2$. As $P_{\mathrm{m}}$ is made very small one would observe a fluid state characterized by a uniform background magnetic field and an azimuthal flow showing deviations from a Keplerian state.

5. The main mode of instability and driver of turbulence in the numerical experiments of the shearing box is the channel mode which is a special response with no radial structure. This mode precipitates a secondary instability which is understood to lead to a turbulent cascade. There is no channel mode allowed in a setup appropriate for a laboratory experiment.

The saturation hypothesis is then the following: is it possible that the fluid response in a shearing sheet environment characterized by very low $P_{\mathrm{m}}$ stabilizes itself by modifying the background shear as suggested by the calculations appropriate for laboratory conditions? Of course, there are some constraints placed upon the way in which the shear may be modified in that the gravitational field predominantly "forces" there to be a Keplerian profile (Ebrahimi et al. 2009). However by analogy to the situation for the laboratory setup, there may come into play undulations of the shear whose average is zero over some length scale $L$ (e.g. Fig. 1).

The main difference between the laboratory setup and numerical calculations of the shearing box are their respective boundary conditions. The numerical experiments adopt periodic boundary conditions while the calculations for the laboratory experiments require no normal flow conditions at an inner and 
outer radius and the former of these permits channel modes to come into play.

Thus, what has been done here is a simple model calculation in which a shearing box configuration with a constant background vertical magnetic field is tested for stability for a number of different shear profiles. The perspective taken is that if there exists a shear profile (say, whose average over some length scale is zero) that is stable to the MRI, then it would strongly suggest that low $P_{\mathrm{m}}$ flow configurations might saturate the MRI by driving into place a new azimuthal flow profile which leads to saturation.

What is found here is something very interesting. For flow configurations in which no-normal flow conditions are imposed at both radial boundaries (Sects. 5.2.1 and 5.6) there exists a modification to the shear in which the most unstable MRI mode is stabilized. However, for flows in which periodic boundary conditions (Sects. 5.2.3 and 5.5) or fixed Lagrangian pressure conditions (Sect. 5.2.2) are imposed there are no modulations of the shear which shut off the instability. In these cases, all modifications of the shear (with zero mean on some radial length scale) seem to enhance instability of the channel mode. For example, if the given channel mode is marginally stable to exponential growth, then introduction of the modified shear, no matter what is its non-zero amplitude, seems to destabilize it as the results of Sects. 5.2.3 and 5.5 indicate.

This strongly implies that in those numerical experiments in which a shear is threaded by a constant vertical magnetic field, the shear modification/MRI-stabilization process may only apply for those flow conditions in which there is no normal flow on either one or both radial boundaries of the system.

On the other hand, for a similar magnetized fluid configuration described by periodic boundaries which therefore permits channel modes to exist, modifications of the shear appears to further destabilize the channel mode. If one were to consider a flow configuration (e.g. the local Keplerian profile in a shearing box threaded by a constant background field) that admits an unstable channel mode, then there seems to be no modification of the shear with zero mean that would saturate the growth of the channel mode since all such modifications further enhances its destabilizing influence. The emergence of a pattern state leading to saturation like the one envisioned by the hypothesis would seem to be out of the question under these circumstances. Moreover this suggests that the tendency for angular momentum transport reduction with $P_{\mathrm{m}}$, as indicated by numerical experiments of the shearing box, is due to some other process or processes (which are not discussed here).

These issues deserve further investigation as the conclusions reached here were done so utilizing very simple functional forms for the shear profile $Q$. Namely, the analysis performed in the previous sections either assumed weak values of $Q$, in order to use singular perturbation theory techniques, or that $Q$ is described by delta-functions (see also below). Use of these functions, which greatly facilitate analysis, have offered some insights into the nature of the responses of these modes. Of course, further investigation would require considering more physically realizable profiles and to check the robustness of the results they yield against the ones offered by use of these more simplified forms. As such the results reached here can serve as guideposts for further enquiries along these lines.

In the following some reflections are presented on other issues pertaining to the implications of the calculations performed here.

\subsection{On the use of delta-functions}

A comparison between the responses of a single delta-function shear profile on an infinite domain (Sect. 5.3) and a top-hat profile for $Q$ (Sect. 5.4) show that the results of the two configurations are qualitatively similar. Although the symmetric step function allows for more normal modes to exist as its radial extent is increased - a feature which is missed if one uses the deltafunction prescription - the qualitative flavor of the response is captured by its use nevertheless.

Another deficiency found in using the defect prescription is that for the top-hat profile studied in Sect. 5.4 there are both even and odd parity normal modes possible whereas only the even-parity mode is admitted in the defect model. The odd-parity mode has no counterpart in the single defect model. It was also shown that the odd-parity mode may exist if the radial domain of the symmetric shear step is large enough as (81) indicates that the minimum size of the domain required for this mode to exist has the proportionality $L \sim 1 / \sqrt{Q_{0}}$. For small values of the defect amplitude the minimum size of the shear step required for this odd-parity mode to exist is so large that one does not expect that this should play any physically relevant role in the dynamics if one is interested in the response to narrow regions of altered shear. It is with some confidence to suppose that it is reasonable to represent the dynamical response of slender regions of modified shear through the use of delta-function defects and this is the motivation and justification for its use in Sects. 5.5 and 5.6.

\subsection{Channel modes}

In the small $Q$ theory of Sect. 5.2, it was shown that channel modes exist for periodic boundary conditions and for conditions in which the total pressure perturbations are zero at the two boundaries. Conversely, if either one of the two boundaries force the velocity perturbation to be zero there, then there is no channel mode permitted as a normal mode solution. In light of the previous results it is important that the boundary conditions appropriate for disks be properly ascertained in order to assess and better understand how activity in them are driven.

Channel modes in the classical limit have no radial structure. In Sect. 5.5 the development of this mode was studied as a function of the defect amplitude $Q_{0}$ and it is shown that though the channel mode develops some amount of radial structure, as evinced by the non-zero value of its wavenumber $\kappa_{00}$, its tendency to be unstable survives and is even enhanced. In Regev \& Umurhan (2008) it was postulated that the channel mode might disappear as a natural mode of the system if some amount of radial symmetry breaking were introduced into the governing equations of motion, for instance, in the form of a radially varying shear profile. However, the results of this section show that the classical channel mode indeed persists in its existence despite the introduction of a shear profile which deviates from the background constant shear state with zero average over some length scale. These conclusions are consistent with similar results regarding the nature of the MRI in more global contexts (Curry et al. 1994).

\subsection{On the existence and number of localized normal modes}

In Sects. 5.3 and 5.4 the existence of localized modes was examined. Localization is understood in this case to correspond to modal disturbances which show exponential decay as the radial coordinate $x \rightarrow \pm \infty$. Thus, by construction, incoming/outgoing 
waves are excluded from consideration ${ }^{8}$. The existence of localized normal modes depends on the relative sign of the shear defect/step profile $Q_{0}$ : HMI modes exist if the shear in the defect/top-hat region is stronger than the background $\left(Q_{0}>0\right)$ while HI modes exist if the shear is relatively weakened $\left(Q_{0}<\right.$ $0)^{9}$ - summarized here as

$$
\begin{aligned}
& Q_{0}>0 \longleftrightarrow \text { hydromagnetic inertial disturbances, } \\
& Q_{0}<0 \longleftrightarrow \text { hydro - inertial disturbances. }
\end{aligned}
$$

Given that the former mode type leads to the MRI, it means that if the shear in the defect zone is weakened, then there are no localized normal modes which can go unstable as only hydroinertial modes are supported (which happen to be stable except for very extreme circumstances). Of course, as an initial value problem, it does not mean that there are no hydromagnetic inertial modes permitted but, instead, the mode probably has some type of algebraic temporal growth/decay associated with it which cannot be described by the usual normal mode approach. This needs to be further examined by studying the associated initial-value problem. Similar features are known to exist for vertically localized MRI modes in disks (Liverts \& Mond 2009) in which the response of the initial value problem can exhibit exponential growth with an amplitude supporting some algebraic time-dependence. Growth can initially be very small and it can take a very long time for a given disturbance to reach the same amplitude as expected for its counterpart normal mode. A conjecture is that the meaning of the absence of normal modes in the problem considered here may have similar attributes to the features associated with the initial-value problem examined by Liverts \& Mond (2009).

Irrespective of this outcome it should be remembered that the dichotomy observed here for modes on an infinite radial domain is a consequence of the imposition of exponential decay of the disturbances. This distinction disappears once waves are allowed to enter and exit from the "infinite" boundaries.

Lastly it is also noteworthy that for the localized problems considered here, when normal modes are permitted they usually come as a finite set. This is in contrast to other localized flow problems considered elsewhere in the literature. For example, in the problem of compact rotating magnetized jets in an infinite medium considered by Bodo et al. (1989) a countably infinite number of normal-modes are predicted for that system. This has some origin in the uniformity of both the rotation and magnetic field profiles of the jet considered in that study. By contrast, there are examples in geophysical flows in which the number of normal mode disturbances are discrete and finite. A recent example can be found in the work of Griffiths (2008) where the stability of inertial waves are studied in a stratified rotating flow with strong horizontal shear. For strongly peaked forms of the potential vorticity only a finite set of normal modes are predicted for the system. Such finiteness of the number of normal modes permitted is not so unusual in systems in which background states, like the shear or stratification, have strongly peaked functional forms like they are in the cases studied in this work.

\footnotetext{
8 Given the mathematical structure of the ode describing axisymmetric disturbances, the radial eigenfunctions that are solutions have either strictly oscillatory or exponential radial profiles. Thus the possibility of decaying oscillations is ruled out.

9 The mode existence dependency upon the sign of $Q_{0}$ was checked for other shear profiles (for instance, by replacing the top-hat with a truncated parabolic profile). Aside from differences in the magnitude of the temporal response and the multiplicity of modes allowed, the aformentioned sign dependency still holds.
}

\subsection{Stabilization in general and an interpretive tool}

The absolute minimum condition that must be met for any normal mode of the system to be unstable in a configuration with constant shear $q_{0}$ is given by the Velikhov criterion (28): if the square of the Alfven frequency $\left(\omega_{a z}^{2}\right)$ is greater than the shear by $2 \Omega_{0}^{2} q_{0}$, then none of the HMI modes of the system can be unstable. For individual modes in the same system with some amount of radial structure the criterion is given by (27), where $\beta_{n}^{2}$ is the square of the radial wavenumber of the disturbance. As can be seen, for fixed values of $\omega_{a z}^{2}$ there are two ways in which stability may be promoted - either by weakening the shear or by increasing the radial wavenumber. In the problem where the shear is constant, these quantities are set from the outset as parameters.

At the end of Sect. 4 there is a series of arguments for arbitrary shear profiles which lead to the identity found in (18), which is like a generalized eigenvalue condition. This relationship is the analog of the dispersion condition of the classical limit (uniform $q$ ) contained in $(25)^{10}$. Care must be adopted before interpreting this expression as an eigenvalue condition since $\sigma$ appears implicitly in the expressions for $\bar{q}$ and $\bar{\beta}$. Nonetheless, a parallel analysis shows that stability is promoted if

$\varpi \equiv \omega_{a z}^{2}-\frac{2 \Omega_{0}^{2} \bar{q} k^{2}}{\bar{\beta}^{2}+k^{2}}>0$,

where the effective wavenumber $\bar{\beta}$ and shear $\bar{q}$, given in $(19,20)$ are,

$\bar{\beta}^{2} \equiv \frac{\int_{\mathcal{D}}\left|\partial_{x} \psi\right|^{2} \mathrm{~d} x}{\int_{\mathcal{D}}|\psi|^{2} \mathrm{~d} x}, \quad \bar{q} \equiv q_{0}+\frac{\int_{\mathcal{D}} Q|\psi|^{2} \mathrm{~d} x}{\int_{\mathcal{D}}|\psi|^{2} \mathrm{~d} x}$

$\mathcal{D}$ is the domain and $\psi$ is the radial eigenmode of the disturbance in question. The stability condition for general $q$ may be rationalized in the same way as the criterion is understood for the classical case with the replacements, $\beta_{n} \rightarrow \bar{\beta}$ and $q_{0} \rightarrow \bar{q}$. With these aforementioned observations and caveats in mind, one may use (92) as an interpretive tool to aid in understanding the reasons for stability/instability in a particular problem. Thus, stability is promoted if (i) for fixed effective wavenumber, the effective shear is reduced or (ii) for fixed effective shear the effective wavenumber is increased, or in more general terms (iii) if the combination results in an overall reduction of the quotient

\section{$\frac{\bar{q}}{\bar{\beta}^{2}+k^{2}}$}

If one considers the results concerning the problem with periodic boundaries in Sect. 5.5, then the persistent instability expected for channel modes can be understood in terms of this criterion. In the classical limit where the defect amplitude is zero then $\bar{\beta}$ for the channel mode is zero. As the defect amplitude is raised $\bar{\beta}$ increases suggesting that this effect has a stabilizing influence, e.g. see Fig. 9. However, as the defect amplitude is raised away from zero the effective shear increases no matter what the sign of $Q_{0}$ and, to effect, this increase always sufficiently overpowers the increase in $\bar{\beta}^{2}$ so that the end result is further instability. In the same figure is plotted the quantity

$$
\frac{\varpi}{\varpi_{0}}=\frac{\bar{q}}{q_{0}} \frac{\beta_{n}^{2}+k^{2}}{\bar{\beta}^{2}+k^{2}},
$$

which, for the generalized Velikhov criterion, is a measure of the ratio of the destabilizing term for the shear profile $q$ compared

\footnotetext{
10 The classical limit is obtained when the replacements $\bar{\beta} \rightarrow \beta_{n}$ and $q \rightarrow q_{0}$ are made.
} 
O. M. Umurhan: Speculation on low magnetic Prandtl number disk velocity profiles

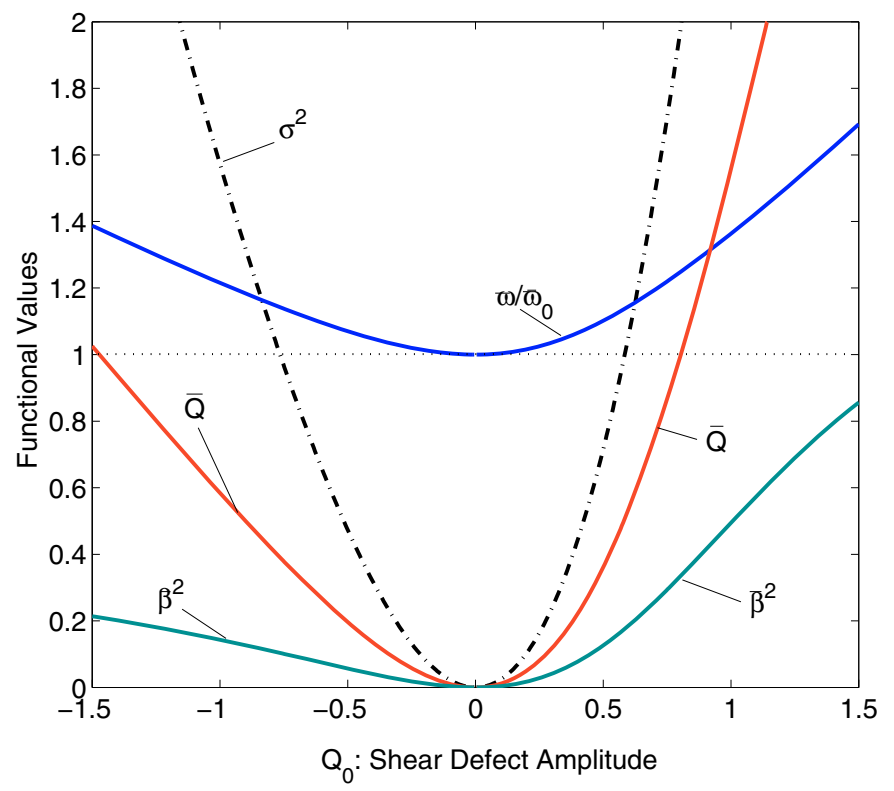

Fig. 9. The behavior of the unstable channel mode for the problem with periodic boundary conditions examined in Sect. 5.5: $k L=\pi$ and $\omega_{a z}^{2}=$ $2 q_{0}$ with $q_{0}=3 / 2$. Plotted is the temporal response, $\sigma^{2}$, together with its corresponding effective wavenumber and shear $\bar{\beta}$ and $\bar{q}=q_{0}+\bar{Q}$ $(\bar{Q}$ plotted $)$, and the stability measure for this mode $\varpi / \varpi_{0}$. All values of the shear defect $Q_{0}$ correspond to increasing the effective shear to above the background value $\bar{Q}\left(Q_{0}\right)>0$. This increase in $\bar{q}$ always overpowers the stabilizing influence of an increased $\bar{\beta}^{2}$. Note, the values of $\sigma^{2}$ have been scaled by an arbitrary factor in order to facilitate clear comparison.

to its nominal value when the shear is only $q_{0}$ : for $\varpi / \varpi_{0}>1$ one expects enhanced destabilization against the classical mode while for $\varpi / \varpi_{0}<1$ comes with enhanced stabilization. In Fig. 9 this quantity is plotted for one of the channel modes discussed in 5.5. Noting that in this case $\beta_{n}=0$ (i.e. that its radial wavenumber is zero in the ideal case) the radial profile of the mode develops as the amplitude of the shear defect increases no matter what its sign. The stabilizing rise in $\bar{\beta}$ is accompanied by the destabilizing increase of the effective shear, that is to say $\bar{Q}>0$, so that the quotient of their respective influences results in $\varpi / \varpi_{0}>1$, indicating instability.

Similarly plotted in Fig. 10 is the influence $Q_{0}$ has upon $\bar{\beta}$, $\bar{q}$, and the stability measure $\varpi / \varpi_{0}$, for the single defect problem on a finite domain studied in Sect. 5.6. The presence of walls filters out the channel mode and the resulting flow potentially can support shear profiles which can stabilize the least stable mode. The Figure shows that within the range of (negative) values of the defect amplitude $Q_{0}$ for which the mode does not exponentially grow/decay the effective shear is less than the background shear state only for part of the stable range. It means to say, then, in that range of parameter values in which the effective shear is greater than the background shear, yet there is still stability of the mode, stabilization is brought through an increased effective radial wavenumber which over-compensates the increased destabilization brought about by an increase in the effective shear.

\subsection{Final reflections and an implication}

The fundamental basis of this study is the assumption that in low magnetic Prandtl number flows for model environments like that considered in this study, the only noticeable outcome of the development of the MRI is to alter the basic shear profile. All other quantities, such as magnetic fields and the radial

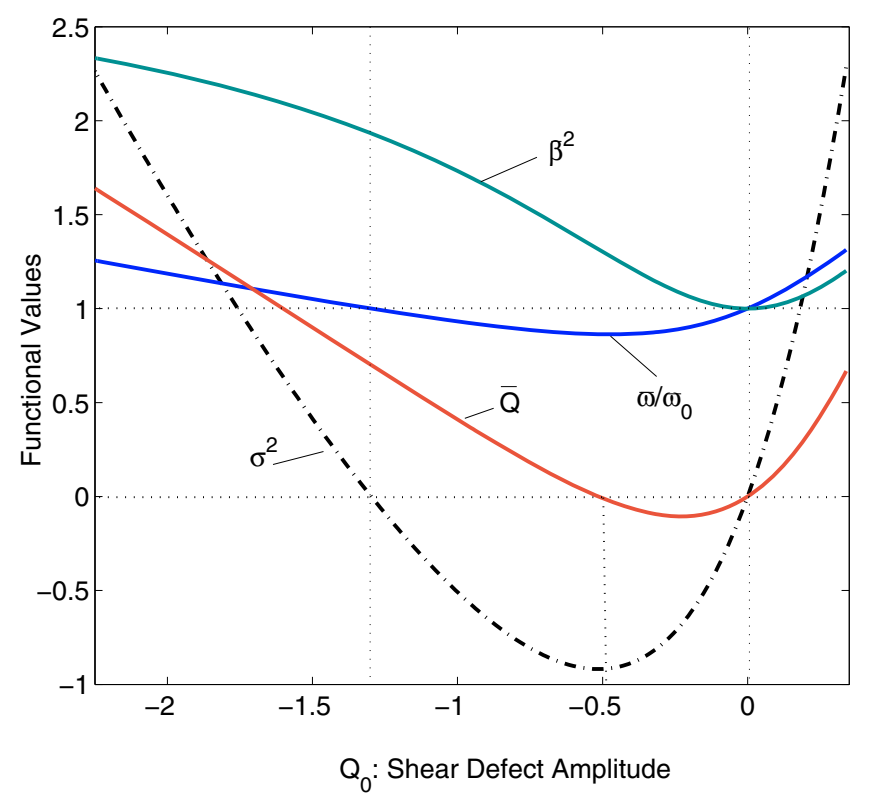

Fig. 10. The behavior of the least stable HMI mode for the problem with channel walls examined in Sect. 5.6: $k L=\pi$ and $\omega_{a z}^{2}=2 q_{0} /\left(k^{2}+\beta_{1}^{2}\right)$ with $\beta_{1}=\pi / L$ and $q_{0}=3 / 2$. Plotted is the temporal response, $\sigma^{2}$, together with its corresponding effective wavenumber and shear $\bar{\beta}$ and $\bar{q}=q_{0}+\bar{Q}(\bar{Q}$ plotted $)$, and the stability measure for this mode $\varpi / \varpi_{0}$. Stability occurs for $Q_{0} \in(-1.32,0)$. In this window the effective shear is less than the background value $\left(\bar{q}-q_{0}=\bar{Q}<0\right)$ for only part of this range, i.e. for $Q_{0} \in(-0.5,0)$. Stabilization in the range $Q_{0} \in(-1.32,-0.5)$ is achieved by a sufficient increase of the effective wavenumber so that the overall effect upon the stabilization parameter $\varpi / \varpi_{0}$ is for it to be less than one. $\sigma^{2}$ has been similarly scaled as in the previous figure.

and vertical velocities, have saturated profiles which scale as some positive power of $P_{\mathrm{m}}$ so that for $P_{\mathrm{m}} \ll 1$ their contributions become negligible. The basis of this assumed trend derives from the theoretical calculations of laboratory setups discussed in the Introduction. It is therefore assumed here that a similar trend may appear for configurations in shearing boxes with periodic radial boundary conditions as well. The perspective taken here with regards to those configurations is that if this is indeed the case, then one can (in principle) test the stability of a wide variety of shear profiles which show deviations (constrained to have a zero average over some length scale) from the basic Keplerian profile - configurations which are akin to those predicted from the calculations of laboratory setups. If a stability calculation shows that there exists a shear profile which is stable to these axisymmetric disturbances, then the shearmodification/mode-saturation hypothesis should become a serious candidate explanation.

However, the calculations done in this study indicate that for periodic shearing box environments there is always an instability of the basic channel mode no matter what shear profile is assumed (satisfying the aforementioned constraints). Unlike the situation for laboratory setups, where saturation can be achieved by altering the basic shear profile, saturation (if present) in shearing box environments with periodic boundary conditions is likely not due to this mechanism.

This further implies that the nonlinear response of systems supporting the MRI stongly depends upon the boundary conditions employed which is not so surprising as many physical systems in Nature exhibit this sensitivity to their boundaries. Thus it seems to this author that some care must be taken before one 
equates the results of laboratory experiments to those physically related/analogous systems for which the experiments are meant to represent (cf. Ji et al. 2006). This cautionary note is not to diminish the value of one over the other, rather, it is intended to emphasize that there are many subtle and, at times, conflicting features between such systems that must be clearly understood before any firm conclusions are reached.

Acknowledgements. The author thanks Marek Abramowicz and the organizers of the Asymptotic Methods in Accretion Disk Theory workshop at CAMK (May/June 2009) where the impetus for this work originated. The author also thanks James Cho, Lancelot Kao, Olivier Gressel, Paola Rebusco, Edgar Knobloch and Oded Regev for their generous support and fruitful conversations during the course of this study.

\section{Appendix A: Proof that $\sigma^{2}$ is never complex}

(15) is rewritten into the form

$\partial_{x}^{2} \psi-k^{2}\left(1+\frac{\omega_{0}^{2}}{\sigma^{2}+\omega_{a z}^{2}}-\frac{4 \Omega_{0}^{2} \omega_{a z}^{2}}{\left(\sigma^{2}+\omega_{a z}^{2}\right)^{2}}-\frac{2 \Omega_{0}^{2} Q}{\sigma^{2}+\omega_{a z}^{2}}\right) \psi=0$,

where $\omega_{0}^{2}=2 \Omega_{0}^{2}\left(2-q_{0}\right)$. The nature of $\sigma^{2}$ will be assessed by supposing that

$\sigma^{2}+\omega_{a z}^{2}=a+i b$

where $a, b \in$ Reals. It will be shown below that $b$ must be zero.

The boundary conditions on $\psi$ are one of the following possibilities: (i) $\psi$ goes to zero on the domain boundary $\partial \mathcal{D}$ provided the latter is finite; (ii) $\psi$ and its derivative go to zero on the domain boundary if the boundary tends to infinite distances; (iii) $\psi$ is periodic on a length scale $L$ in the domain $\mathcal{D}$. Replacing $\sigma^{2}+\omega_{a z}^{2}$ with $a+i b$ in the equation for $\psi$, followed by multiplication by the complex conjugate of $\psi\left(\right.$ i.e. $\left.\psi^{*}\right)$, integrating the result and applying the boundary conditions gives

$I_{1}+i I_{2}=0$

where

$$
\begin{aligned}
I_{1}= & -\int_{\mathcal{D}}\left|\partial_{x} \psi\right|^{2} \mathrm{~d} x \\
& -k^{2} \int_{\mathcal{D}}\left[1+a \frac{\omega_{0}^{2}-2 \Omega_{0}^{2} Q}{a^{2}+b^{2}}-4 \Omega^{2} \omega_{a z}^{2} \frac{a^{2}-b^{2}}{a^{2}+b^{2}}\right]|\psi|^{2} \mathrm{~d} x,
\end{aligned}
$$

and

$I_{2}=b k^{2} \int_{\mathcal{D}}\left[\frac{\omega_{0}^{2}-2 \Omega_{0}^{2} Q}{a^{2}+b^{2}}-\frac{4 \Omega_{0}^{2} \omega_{a z}^{2} 2 a}{\left(a^{2}+b^{2}\right)^{2}}\right]|\psi|^{2} \mathrm{~d} x$.
It must be that both $I_{1}$ and $I_{2}$ are zero if (A.1) is to be satisfied. Supposing that $b \neq 0$ then the relationship implied by $I_{2}=0$, is that

$\int_{\mathcal{D}}\left[\frac{\omega_{0}^{2}-2 \Omega_{0}^{2} Q}{a^{2}+b^{2}}\right]|\psi|^{2} \mathrm{~d} x=\int_{\mathcal{D}}\left[\frac{4 \Omega_{0}^{2} \omega_{a z}^{2} 2 a}{\left(a^{2}+b^{2}\right)^{2}}\right]|\psi|^{2} \mathrm{~d} x$

This result used in the expression for $I_{1}$ lead to

$I_{1}=-\int_{\mathcal{D}}\left|\partial_{x} \psi\right|^{2} \mathrm{~d} x-k^{2}\left[1+\frac{4 \Omega^{2} \omega_{a z}^{2}}{a^{2}+b^{2}}\right] \int_{\mathcal{D}}|\psi|^{2} \mathrm{~d} x$.

However all the quantities in the above expression are always positive and, therefore, $I_{1}$ can never be zero. Consequently $b$ must be zero which, in turn, means that $\sigma^{2}$ can only be real.

With $b=0$ and restoring the definition of $a$ the equation $I_{1}=0$ appears now as

$$
\begin{aligned}
0= & -\int_{\mathcal{D}}\left|\partial_{x} \psi\right|^{2} \mathrm{~d} x \\
& -k^{2} \int_{\mathcal{D}}\left[1+\frac{\omega_{0}^{2}-2 \Omega_{0}^{2} Q}{\sigma^{2}+\omega_{a z}^{2}}-\frac{4 \Omega^{2} \omega_{a z}^{2}}{\left(\sigma^{2}+\omega_{a z}^{2}\right)^{2}}\right]|\psi|^{2} \mathrm{~d} x .
\end{aligned}
$$

Dividing the above expression by $\int_{\mathcal{D}}|\psi|^{2} \mathrm{~d} x$ and utilizing the definition of $\kappa_{0}^{2}$ given in (22) leads to the expressions appearing in $(18-20)$.

\section{References}

Acheson, D. J., \& Hide, R. 1973, Rep. Prog. Phys., 36, 159

Balbus, S. A., \& Hawley, J. F. 1991, ApJ, 376, 214

Balbus, S. A., \& Henri, P. 2008, ApJ, 674, 408

Bender, C. M., \& Orszag, O. 1999, Advanced Mathematical Methods for Scientists and Engineers (Springer)

Bodo, G., Rosner, R., Ferrari, A., \& Kobloch, E. 1989, ApJ, 341, 631

Chandrasekhar, S. 1961, Hydrodynamic and Hydromagnetic Stability (Oxford)

Curry, C., Pudritz, R. E., \& Sutherland, P. G. 1994, ApJ, 434, 206

Ebrahimi, F., Prager, S. C., \& Schnack, D. D. 2009, ApJ, 698, 233

Friedman, B. 1956, Principles and Techniques of Applied Mathematics (New York: Wiley)

Fromang, S., Papaloizou, J., Lesur, G., \& Heinemann, T. 2007, A\&A, 476, 1123

Goldreich, P., \& Lynden-Bell, D. 1965, MNRAS, 130, 125

Griffiths, S. D. 2008, J. Fluid Mech., 605, 115

Jamroz, B., Julien, K., \& Knobloch, E. 2008, Astron. Nachr., 329, 675

Ji, H., Burin, M., Schartman, E., \& Goodman, J. 2006, Nature, 444, 343

Julien, K., \& Knobloch, E. 2006, in Stellar Fluid Dynamics and Numerical

Simulations: From the Sun to Neutron Stars, ed. M. Rieutord, \& B. Dubrulle, EAS Publ. Ser., 21, 81

Knobloch, E. 1992, MNRAS, 255, 25

Knobloch, E., \& Julien, K. 2005, Phys. Fluids, 17, 094106/16

Lesur, G., \& Longaretti, P.-Y. 2007, MNRAS, 378, 1471

Liverts, E., \& Mond, M. 2009, MNRAS, 392, 287

Regev, O., \& Umurhan, O. M. 2008, A\&A, 481, 1

Umurhan, O. M., Regev, O., \& Menou, K. 2007a, PRL, 98, 034501

Umurhan, O. M., Menou, K., \& Regev, O. 2007b, PRE, 76, 036310

Velikhov, E. P. 1959, J. Exp. Theor. Phys. (USSR), 36, 995 